ARTICLE

Received 23 Mar 2014 | Accepted 14 Apr 2014 | Published 23 May 2014

DOI: $10.1038 /$ ncomms4891

OPEN

\title{
Clathrin light chains are required for the gyrating-clathrin recycling pathway and thereby promote cell migration
}

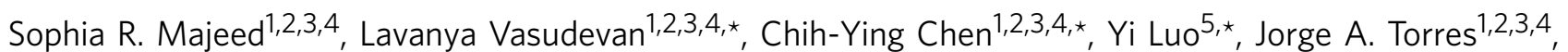
Timothy M. Evans 1,2,3,4, Andrew Sharkey6 ${ }^{6}$, Amy B. Foraker1,2,3,4, Nicole M.L. Wong1,2,3,4, Christopher Esk ${ }^{1,2,3,4}$, Theresa A. Freeman ${ }^{7}$, Ashley Moffett ${ }^{6}$, James H. Keen ${ }^{5}$ \& Frances M. Brodsky ${ }^{1,2,3,4}$

The clathrin light chain (CLC) subunits participate in several membrane traffic pathways involving both clathrin and actin, through binding the actin-organizing huntingtin-interacting proteins (Hip). However, CLCs are dispensable for clathrin-mediated endocytosis of many cargoes. Here we observe that CLC depletion affects cell migration through Hip binding and reduces surface expression of $\beta 1$-integrin by interference with recycling following normal endocytosis of inactive $\beta 1$-integrin. CLC depletion and expression of a modified CLC also inhibit the appearance of gyrating $(G)$-clathrin structures, known mediators of rapid recycling of transferrin receptor from endosomes. Expression of the modified CLC reduces $\beta 1$-integrin and transferrin receptor recycling, as well as cell migration, implicating G-clathrin in these processes. Supporting a physiological role for CLC in migration, the CLCb isoform of CLC is upregulated in migratory human trophoblast cells during uterine invasion. Together, these studies establish CLCs as mediating clathrin-actin interactions needed for recycling by G-clathrin during migration.

\footnotetext{
${ }^{1}$ Department of Bioengineering and Therapeutic Sciences, University of California San Francisco, San Francisco, California 94143, USA. ${ }^{2}$ Department of Pharmaceutical Chemistry, University of California San Francisco, San Francisco, California 94143, USA. ${ }^{3}$ Department of Microbiology and Immunology, University of California San Francisco, San Francisco, California 94143, USA. ${ }^{4}$ The G.W. Hooper Foundation, University of California San Francisco, San Francisco, California 94143, USA. ${ }^{5}$ Department of Biochemistry and Molecular Biology, Thomas Jefferson University, Philadelphia, Pennsylvania 19107 , USA. ${ }^{6}$ Department of Pathology and Centre for Trophoblast Research, University of Cambridge, Cambridge CB2 1QP, UK. ${ }^{7}$ Department of Orthopedic Surgery, Thomas Jefferson University, Philadelphia, Pennsylvania 19107, USA. * These authors contributed equally to this work. Correspondence and requests for materials should be addressed to F.M.B. (email: Frances.Brodsky@ucsf.edu).
} 
C lathrin plays a key role in intracellular membrane traffic by polymerizing into a membrane-associated latticed coat that captures cargo during receptor-mediated endocytosis and organelle biogenesis ${ }^{1}$. The lattice-forming clathrin triskelion is composed of trimerized clathrin heavy chain (CHC) subunits, which comprise the determinants for self-assembly. The major $\mathrm{CHC}$ isoform $(\mathrm{CHC17})$ is bound by clathrin light chain (CLC) subunits that extend half way along the triskelion leg. There are two CLCs in vertebrates (CLCa and $\mathrm{CLCb}$ ) with characteristic tissue-specific expression. Though their cellular functions have yet to be fully defined, CLCs stabilize CHC17 trimerization ${ }^{2}$ and regulate lattice formation in vitro ${ }^{3}$. Although depletion of $\mathrm{CHC} 17$ globally impairs clathrin-mediated endocytosis $(\mathrm{CME})^{4-6}$, the only known cargo affected by CLC depletion are certain G-protein-coupled receptors ${ }^{7}$. Uptake of transferrin receptor, epidermal growth factor (EGF) receptor, cation-independent-mannose-6-phosphate receptor and lowdensity lipoprotein receptor occur in the absence of $\mathrm{CLC}^{5,6}$. However, depletion of CLC effectively inhibits internalization of cargo such as bacteria and virus particles that are too large for conventional CME but require clathrin and actin for uptake $e^{8,9}$. CLCs are also required for internalization from villous membranes under tension on mammalian cells and from the yeast plasma membrane under turgor pressure, processes that likewise require actin ${ }^{10-12}$. These actin-dependent clathrin pathways involve the actin-binding proteins huntingtininteracting protein 1 (Hip1), its related homologue Hip1R or Sla2p in yeast, all of which bind to $\mathrm{CLCs}^{1}$. The 22-residue Hip-binding region is the only sequence completely shared by CLCa and CLCb and is conserved, but not identical, in yeast CLC, suggesting a key biological function ${ }^{13-16}$.

Cell migration represents a different process involving coordination of clathrin and actin activity. Migration requires trafficking of the integrins that bind extracellular matrix ${ }^{17-20}$ through endocytic, as well as fast and slow recycling pathways ${ }^{17-19,21}$. Clathrin has been implicated in cell migration, as siRNA targeting $\mathrm{CHC17}$ or CLC overexpression affect cell motility ${ }^{18,22-24}$. However, distinct roles for CHC and CLC were not addressed in these studies. The rapid recycling pathways for integrins during cell migration depend on actin and actin-modulating proteins ${ }^{25}$. Rapid endosomal recycling ${ }^{26,27}$ can be mediated by intracellular peripheral clathrin-coated structures termed 'gyrating-clathrin' (G-clathrin), characterized by live cell imaging of fluorescent CLC or GGA1 adaptors ${ }^{26,28}$. The potential coincidence of these pathways inspired the investigation of a role for the CLC-actin connection during the rapid recycling that occurs in cell migration that we report here.

We observe that complete loss of both clathrin subunits (by CHC17 depletion) or of CLC alone impedes cell migration, but resulting actin morphologies and focal adhesion characteristics suggest distinct roles for the clathrin subunits. Analysis of integrin trafficking reveals that CLC-Hip interactions are needed for recycling of inactive $\beta 1$-integrin, whereas $\mathrm{CHC} 17$ but not CLC is needed for its internalization. Furthermore, disruption of CLC function significantly reduces G-clathrin structures marked by Golgi-localized gamma-adaptin ear containing Arf-binding protein 1 (GGA1), and attenuates transferrin receptor recycling. In support of a key role for CLC in cell migration, we observe upregulation of $\mathrm{CLCb}$, along with other proteins involved in migration and adhesion, in trophoblast cells that invade the uterus during fetal implantation. Our combined results establish that the clathrin-actin interactions mediated by the CLC subunits are important for G-clathrin recycling of $\beta 1$-integrin during cell migration and have potential significance for Hip1 upregulation in metastatic cancers.

\section{Results}

Actin and focal adhesions differ upon CLC or CHC depletion. Following up our published observation that overexpression of the Hip-binding CLC fragment (residues 1-44) dramatically alters cortical cellular actin distribution ${ }^{13}$, we characterized the effects of CLC depletion on actin organization at the cell periphery. Cells were depleted of CLCs using siRNA targeting both CLCa and CLCb, or of total clathrin using siRNA targeting $\mathrm{CHC17}$. During depletion, cells were co-transfected with either mutant CLCa (I38A, D25A) that does not bind Hip proteins but does bind $\mathrm{CHC17}$ (ref. 13) or with WT CLCa (both siRNAresistant and HA-tagged), or with the empty expression vector. Consistent with previous studies, CLC depletion generated short, disorganized actin structures characterized by the presence of cortactin at the tips ${ }^{6}$. This phenotype was rescued by WT CLCa, but not by mutant CLCa (Fig. 1a). CHC17-depleted cells had a completely different actin phenotype characterized by increased long actin fibres at the cell periphery. This phenotype was not reversed by expression of CLCs, which depend on $\mathrm{CHC17}$ for membrane recruitment. No phenotype was seen in cells expressing the mutant CLCa without endogenous CLC depletion. These results show distinct effects of $\mathrm{CHC17}$ and CLC depletion on actin morphology and extend our previous findings that CLC's influence on actin is mediated through Hip binding.

The changes in actin upon depletion of either clathrin subunit suggested potential correlative changes in focal adhesions resulting from these perturbations. Compared with controltreated cells, bright patches stained for the focal adhesion marker paxillin were more obvious in CHC17-depleted cells, whereas paxillin patches appeared duller and were reduced in CLCdepleted cells (Fig. 1b). Quantitative analysis revealed that $32 \%$ of the cell periphery in $\mathrm{CHC} 17$-depleted cells was occupied with paxillin-containing focal adhesions, compared with $17 \%$ of control and less than $10 \%$ of CLC-depleted cells (Fig. 1c). Thus, our data suggest that CLCs play a unique role in influencing focal adhesion morphology distinct from the pathway affected by depletion of both clathrin heavy and light chain subunits upon CHC17 targeting (Fig. 1d).

Loss of CLC-Hip coupling impairs cell migration. Clathrin has been implicated in cell migration ${ }^{18,22-24,29}$ and this has been attributed to a role in endocytosis at focal adhesions, a role in plaque formation and SCAR-WAVE binding by $\mathrm{CHC} 17$. Although CLC depletion has variable effects on endocytosis ${ }^{5-7}$, our observations (Fig. 1) that CLC influences actin and focal adhesions led us to address the role of CLC in cell migration. HeLa cells depleted of CLC or CHC17 were grown to confluency and migration was assessed in a wound-healing assay. Depletion of $\mathrm{CHC17}$ impaired HeLa cell migration as measured by displacement by $35 \%$ relative to control-treated cells (Fig. $2 \mathrm{a}-\mathrm{c}$ ), consistent with previous reports ${ }^{18,24}$ without affecting cell speed. Migration of a HeLa cell derivative expressing SNAP-tagged $\mathrm{CLCa}^{30}$, in which whole clathrin was acutely inactivated by drug-induced crosslinking of the SNAP tag, was similarly impaired (Supplementary Fig. 1a). Notably, CLC depletion reduced HeLa cell migratory displacement by $22 \%$, also without affecting speed (Fig. 2a-c). Depletion of the second $\mathrm{CHC}$ isoform $\mathrm{CHC} 22$, which does not influence CLC or CHC17 levels or participate in endocytosis ${ }^{31,32}$ had no effect on HeLa cell migration (Supplementary Fig. 1b,c). Cell proliferation was not significantly altered by siRNA depletion of either clathrin subunit $24-48 \mathrm{~h}$ or by clathrin inactivation post cell plating, indicating that wound-healing defects could be ascribed directly to altered migration (Supplementary Fig. 2a-c).

In H1299 lung cancer cells, which migrate faster than HeLa cells, $\mathrm{CHC} 17$ depletion only reduced migration by $17 \%$, whereas 
a

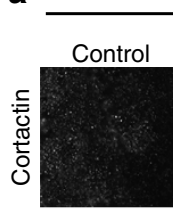

+ Vector

竞
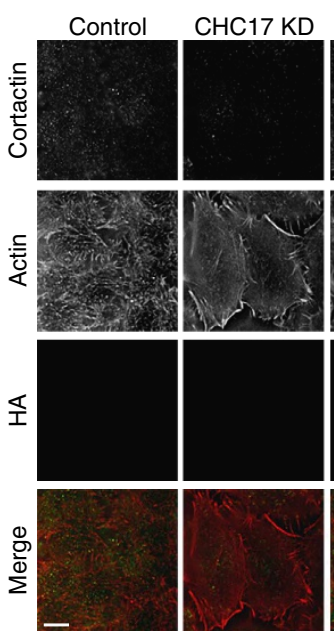

b
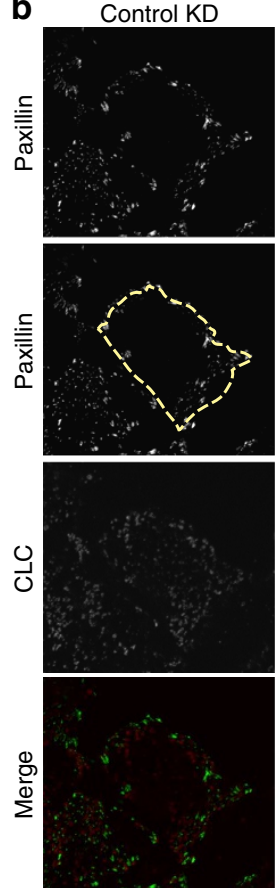

$\mathrm{CHC} 17 \mathrm{KD}$
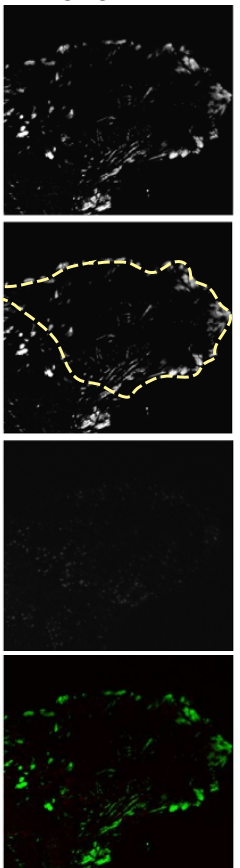

$+\mathrm{CLCa}$
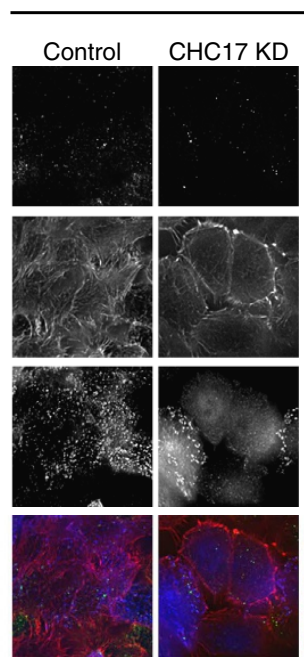

CLCab KD
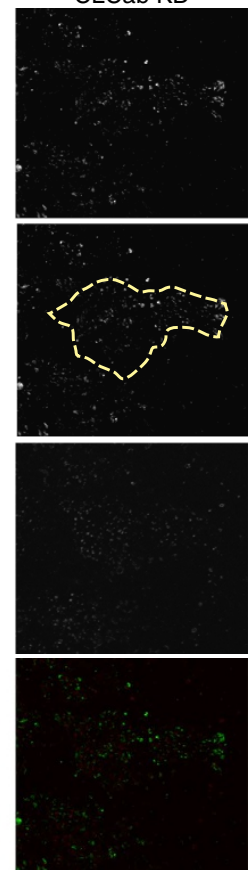

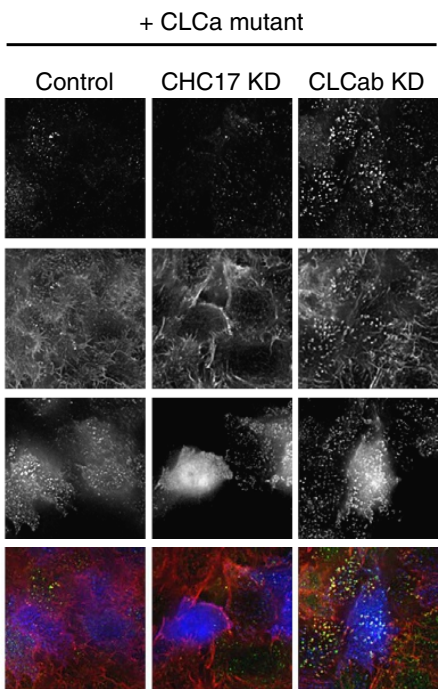

C

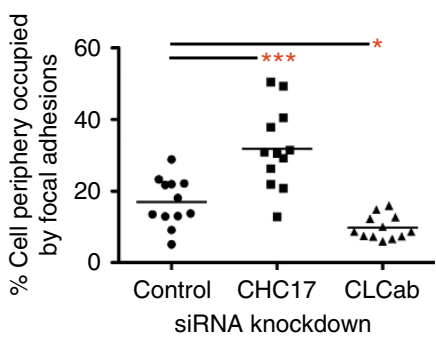

d

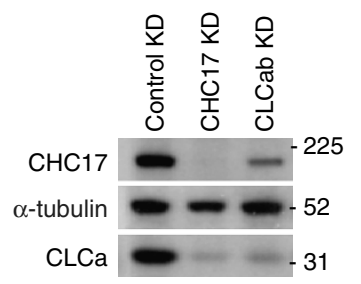

Figure 1 | CLC-depleted HeLa cells display disorganized actin fibres and reduced focal adhesions. (a) HeLa cells treated with siRNA against CHC17, CLCab or control siRNA and transiently transfected with siRNA-resistant HA-tagged-CLCa or HA-tagged-CLCa mutant were labelled for cortactin (green in merge), actin (red) and HA (blue). In all the panels, the overlap of green and red signals is shown in yellow, scale bars, $10 \mu \mathrm{m}$. (b) HeLa cells transfected with siRNAs against CHC17, CLCab or control siRNA were stained for paxillin (green in merge) and CLC (red). The cell border is outlined in the second panel from the top (yellow dashed line). (c) The cell periphery occupied by focal adhesions of cells in $\mathbf{b}$ was measured using Image $\mathrm{J}$ to outline cells and quantify paxillin staining at the border (mean; $n=12$ cells from two independent experiments; ${ }^{\star} P<0.05$, ${ }^{\star \star \star} P<0.005$, one-way analysis of variance followed by Newman-Keuls post hoc test). (d) HeLa cells were treated with the indicated siRNA for $72 \mathrm{~h}$, harvested and subjected to immunoblotting analysis. Control, scrambled siRNA; KD, knockdown. A representative blot of many experiments is shown. Migration positions of molecular mass markers are indicated in $\mathrm{kDa}$ at the right of the immunoblots shown.

CLC depletion reduced migration by $41 \%$ relative to control cells (Fig. 2d-f), validating that the contribution of CLC to cell migration is independent of the associated decrease in $\mathrm{CHC} 17$ stability upon CLC depletion. As seen for HeLa, CHC or CLC depletion mainly affected H1299 cell displacement, though double depletion also affected speed (Fig. 2f) and CHC22 depletion had no effect (Supplementary Fig. 1d,e). However, Hip depletion from H1299 cells decreased cell migration by $27 \%$ (Fig. 2d-f), while having a marginal effect on HeLa cell migration. This suggests that CLC-Hip protein interaction plays a greater role in regulation of cell migration in $\mathrm{H} 1299$ cells compared with HeLa cells. Interestingly, H1299 cells express more Hip1 protein relative to $\mathrm{CHC17}$ than HeLa cells.

To address whether the role of CLC in migration requires association with Hip proteins, we generated H1299 and HeLa cell clones stably expressing siRNA-resistant mutant CLCa or CLCb that do not bind Hip proteins ${ }^{13}$ or siRNA-resistant WT CLCa or $\mathrm{CLCb}$, using the non-coding vector as a control. Each stable transfectant was depleted of endogenous CLCa and CLCb or treated with control siRNA, and migration was compared 
a
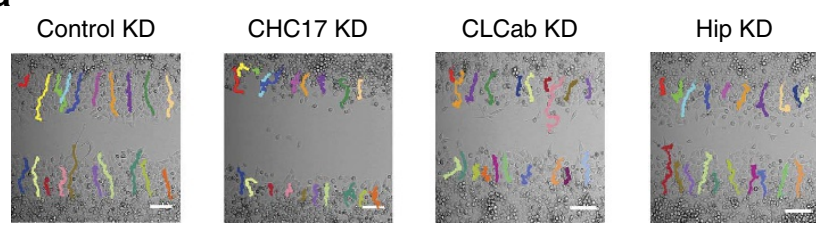

b
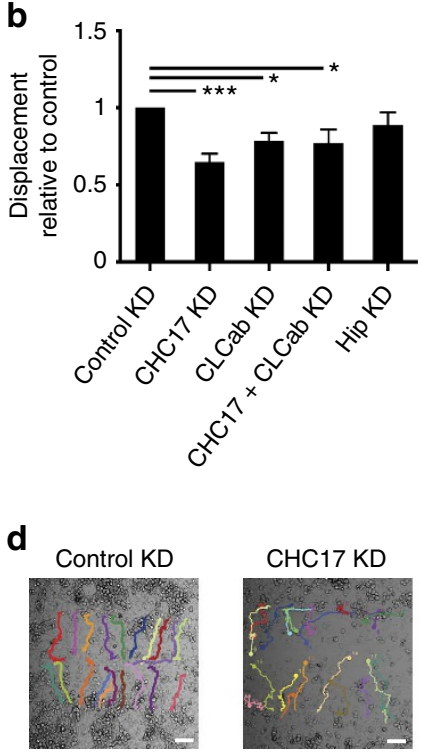

f

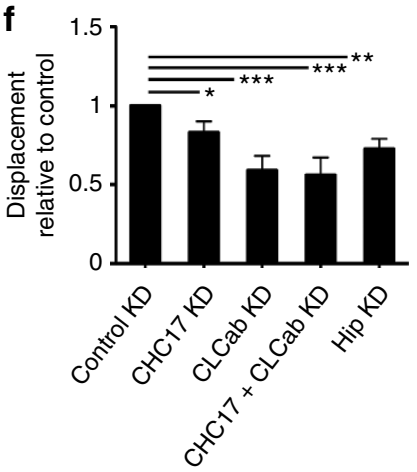

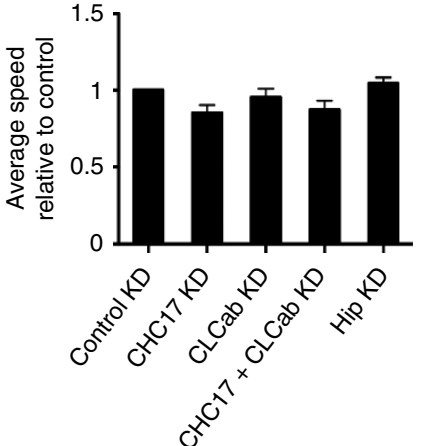

Hip KD
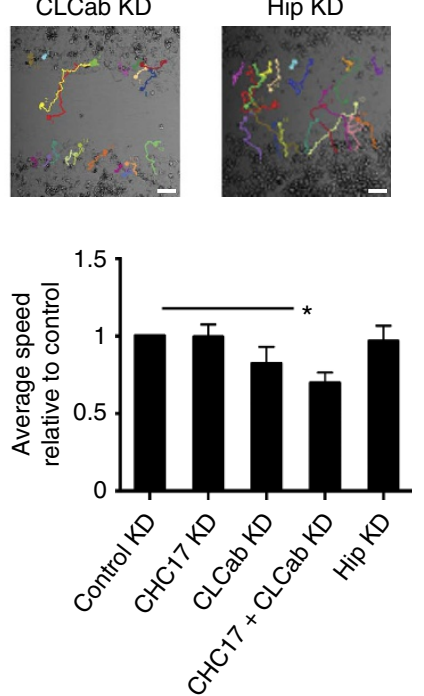

C

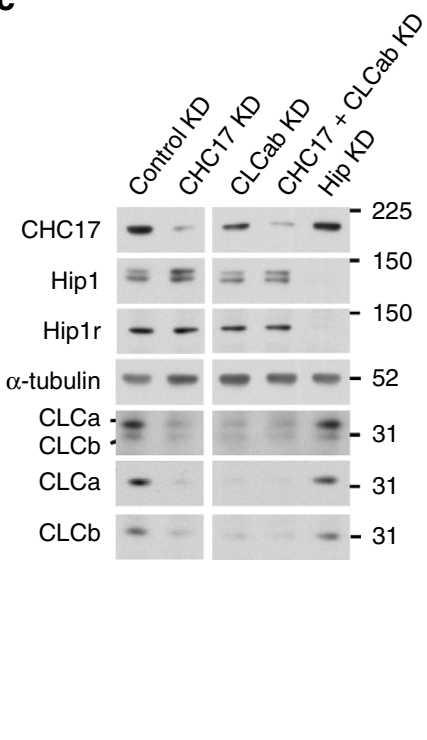

e

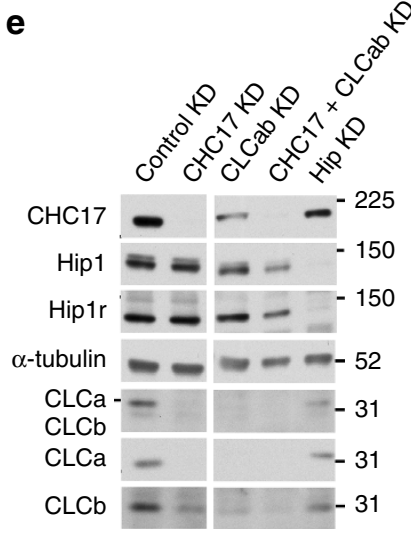

Figure 2 | CHC17 or CLC depletion decreases HeLa and H1299 cell migration. Wound-healing assays were performed in cells transfected with siRNA against $\mathrm{CHC17}, \mathrm{CLCab}$, Hip (Hip1 and Hip1R) or control siRNA. Migration across the wound was imaged in the presence of medium containing $1 \%$ serum on glass-bottomed plates using live-cell time-lapse microscopy. (a) Representative HeLa cell trajectories at end time points (24 h) are shown. The MtrackJ plugin of ImageJ was used to manually trace migratory cell tracks, marked in colour. (b) Quantitative analysis of HeLa cell relative net displacement (net displacement from the origin relative to control; left) and average speed (distance migrated per min relative to control; right) were quantified from migratory tracks (mean \pm s.e.m. of at least 230 cells analysed from 11 independent experiments; as in a. ${ }^{\star} P<0.05$, ${ }^{\star \star \star} P<0.005$, one-way analysis of variance (ANOVA) followed by Newman-Keuls post hoc test). (c) Representative immunoblots of siRNA treatments of HeLa cells as in $\mathbf{a}$.

(d) H1299 cell trajectories at end time points ( $15 \mathrm{~h}$ ) are shown. The MtrackJ plugin of ImageJ was used to manually trace cell tracks as in a

(e) Representative immunoblots of siRNA treatments as in d. (f) Quantitative analysis of $\mathrm{H} 1299$ cell relative net displacement (left) and average speed (right) from migratory tracks (mean \pm s.e.m. of at least 100 cells analysed from five independent experiments; as in $\mathbf{d} .{ }^{\star} P<0.05,{ }^{\star \star} P<0.01,{ }^{\star \star \star} P<0.005$, one-way ANOVA followed by Newman-Keuls post hoc test). Migration positions of molecular mass markers are indicated in kDa at the right of the immunoblots shown. Scale bars, $100 \mu \mathrm{m}$.

between these two conditions for each clone. Migration defects following CLC depletion were obvious in H1299 clones with siRNA-resistant mutant CLCa or CLCb but did not occur in the clones expressing WT CLCa or WT CLCb (Fig. 3). These results were replicated in HeLa cell lines stably expressing siRNAresistant WT or Hip-binding-deficient $\mathrm{CLCb}$ (Supplementary Fig. 3). We were unable to establish HeLa clones that stably express WT CLCa or mutant CLCa. These data demonstrate that CLC plays a role in cell migration that requires its binding to Hip.
Migration defects caused by depletion of both CLCs were rescued by expression of WT CLCa or WT CLCb, indicating that the role in migration is a shared property of the CLC isoforms, consistent with their identical Hip-binding sequences ${ }^{13}$.

Inactive B1-integrin recycling involves CLC and G-clathrin. Focal adhesions and cell migration are affected by surface integrin levels ${ }^{17}$, so we assessed if these were altered by CLC depletion using cell surface biotinylation. We focused on $\beta 1$-integrin, the 

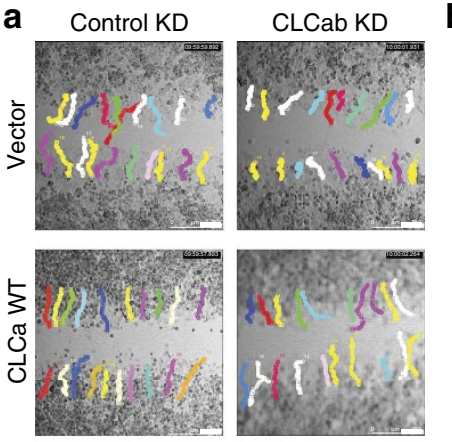

b
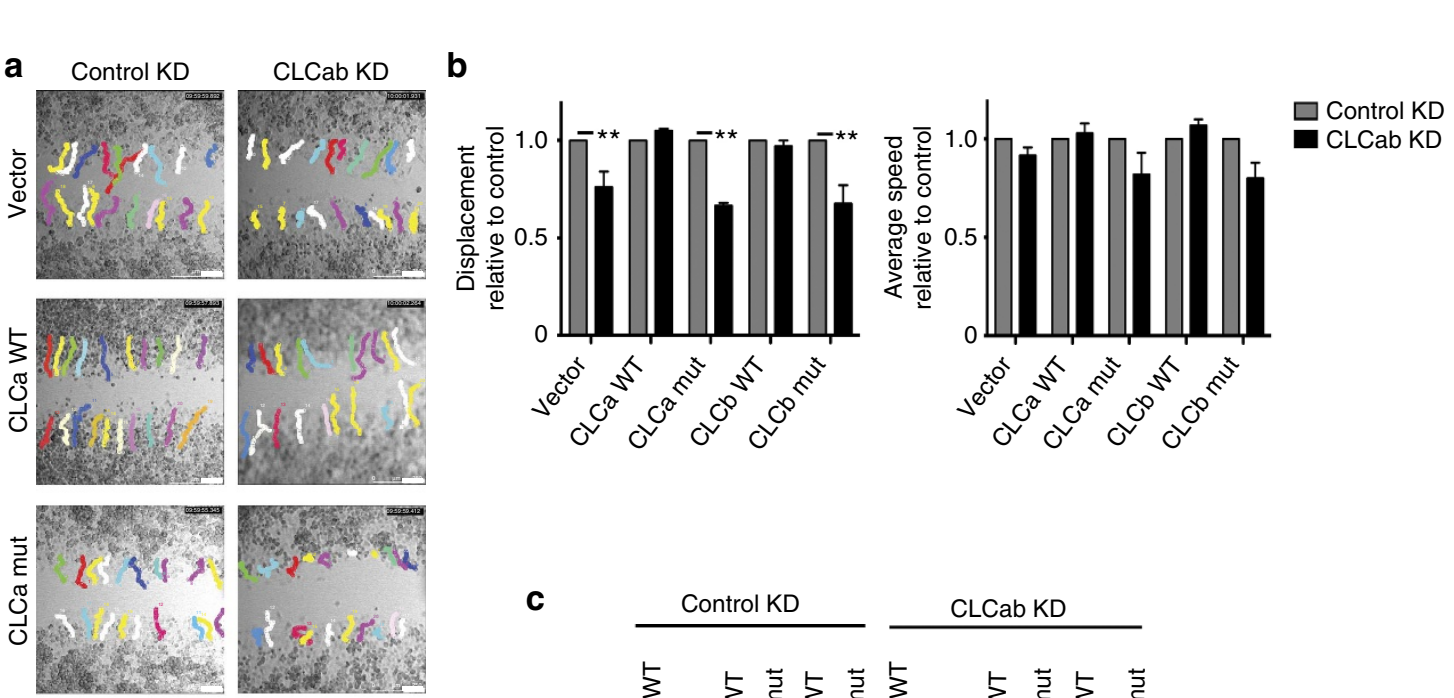

c
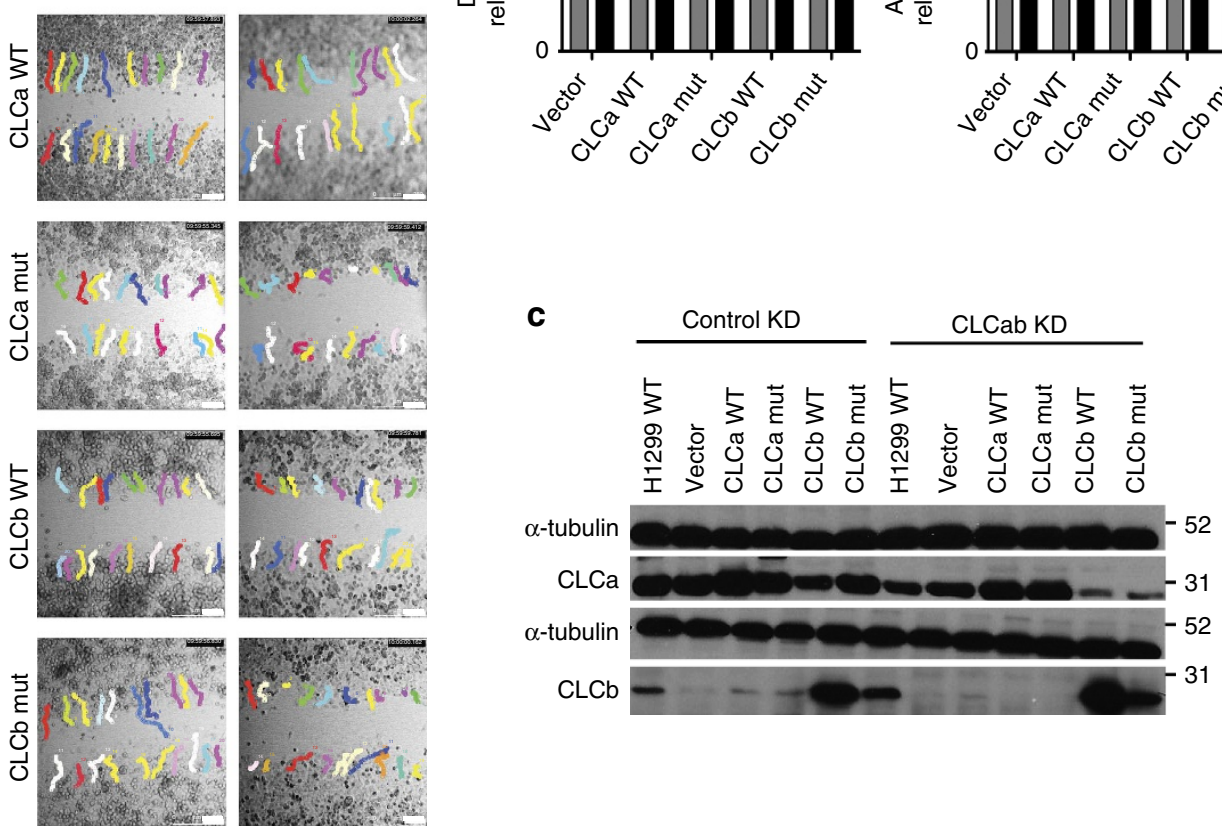

\section{LE}


a
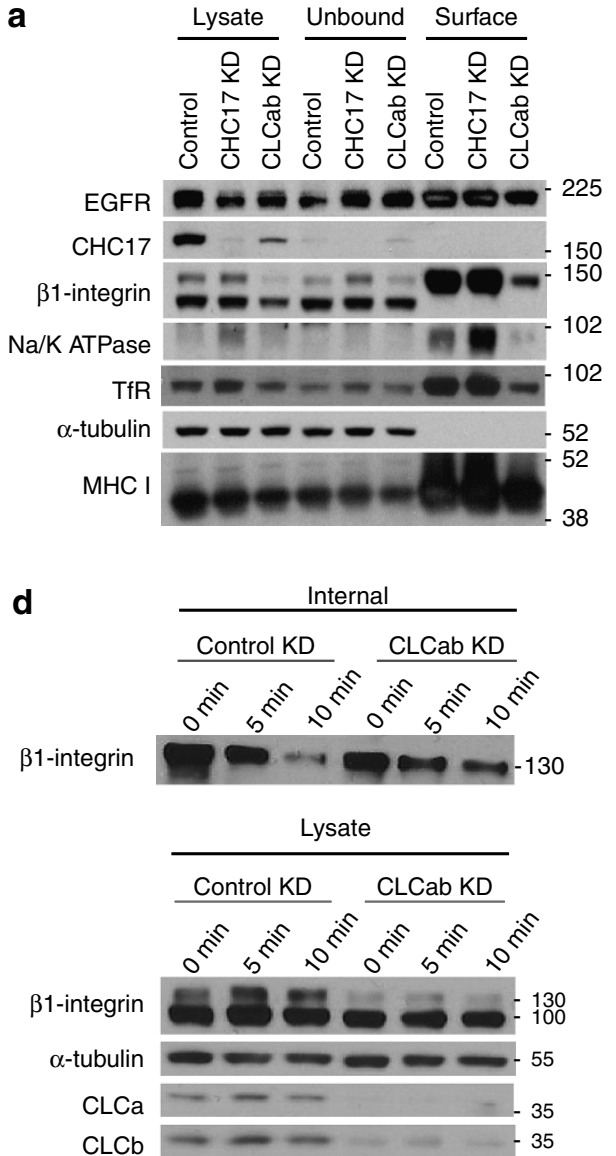

b

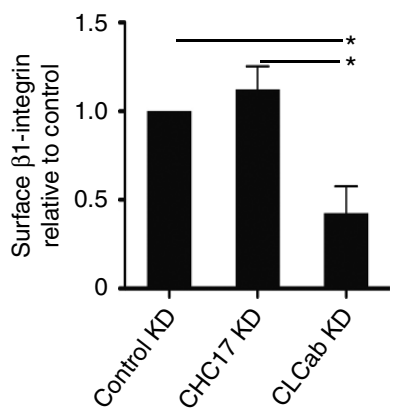

C

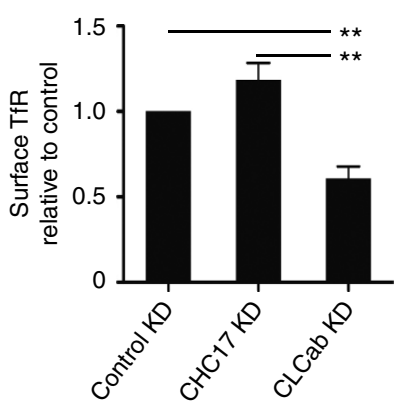

e

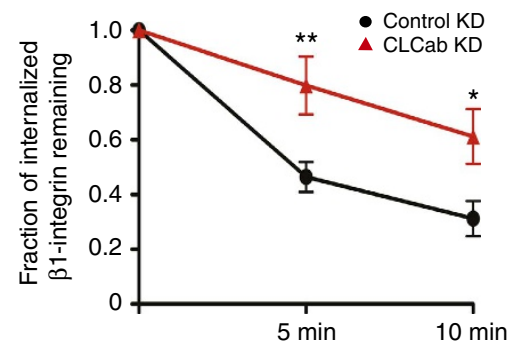

Figure 4 | CLC depletion reduces constitutive recycling of $\boldsymbol{\beta} 1$-integrin and steady state levels of other surface molecules. (a) HeLa cells transfected with the indicated siRNAs for knockdown (KD) were biotinylated for 30 min and surface biotinylated proteins were isolated using streptavidin beads, followed by immunoblotting for the indicated proteins. (b,c) Quantification of surface levels of (b) $\beta 1$-integrin and (c) transferrin receptor (TfR) from $\mathbf{a}$ and replicate experiments (mean \pm s.e.m.; $n=3$; ${ }^{\star} P<0.05$, ${ }^{\star \star} P<0.01$, one-way analysis of variance (ANOVA) followed by Newman-Keuls post hoc test). (d) Recycling of internalized $\beta 1$-integrin in control and CLCab-siRNA-treated HeLa cells. Cells were biotinylated and allowed to internalize surface proteins for 30 min at $37^{\circ} \mathrm{C}$. Surface biotin was removed by reduction and internalized proteins were chased back to the cell surface for the indicated times at $37^{\circ} \mathrm{C}$. Surface biotin was reduced again, and cells were lysed. Above: Biotinylated proteins were bound to streptavidin beads, followed by immunoblotting for $\beta 1$-integrin. Below: Immunoblots of indicated proteins in total cell lysate at each time point. Representative immunoblots of one experiment $(n=6)$. (e) Quantification of $\beta 1$-integrin recycling assays as in $\mathbf{d}$ (mean \pm s.e.m.; $n=6$; ${ }^{\star} P<0.05$, ${ }^{\star \star} P<0.01$, two-way ANOVA followed by Bonferroni post hoc test). Migration positions of molecular mass markers are indicated in $\mathrm{kDa}$ at the right of the immunoblots shown.

Following depletion of endogenous CLCs from HeLa clones expressing WT or mutant CLCb (Fig. 5c), antibody to inactive $\beta 1$-integrin was internalized (Fig. 5d). A recycling defect was observed for cells expressing the mutant CLCb but rescued in cells expressing WT CLCb (Fig. 5e). Thus, recycling of inactive $\beta 1$-integrin involves CLC-Hip interactions. Internalization of inactive $\beta 1$-integrin was partially impaired in the cells expressing the mutant CLCb (Fig. 5d), but internalized antibody was not recycled in these cells upon depletion of endogenous CLC.

Integrins transit through rapid and slow recycling pathways during cell migration. A role for clathrin in recycling to the plasma membrane from endosomes has been much debated, but it is clear that one of the pathways for rapid recycling of transferrin receptor involves G-clathrin ${ }^{26}$. G-clathrin has been visualized using fluorescent protein fusions with CLCs or with GGA1 adaptors, with which it is associated in the cell periphery ${ }^{26,28}$, and its recruitment to endosome-derived structures is ADP-ribosylation factor 6 (ARF6)-dependent ${ }^{34}$. Many ARF6-dependent pathways involve actin, thus suggesting a role for CLCs in G-clathrin. To visualize G-clathrin under CLC depletion conditions, HeLa-M cells (a flat subclone of HeLa cells) were simultaneously transfected with siRNA and GGA1 coupled to yellow fluorescent protein (YFP) ${ }^{26,34}$. Live transfected cells were imaged using continuous $30 \mathrm{~ms}$ exposures (Supplementary Movie 1) and it was obvious that the peripheral, highly mobile GGA1-containing structures were significantly reduced in cells treated with siRNA targeting CLC. These structures were also absent from cells co-transfected with CLCa coupled to DsRed1 (CLCa-DsRed1). GGA1 in G-clathrin structures was quantified by comparing segmented maximum projection images (comprising both highly mobile G-clathrin and stable clathrin structures) to summed projection images (emphasizes relatively stationary structures, not G-clathrin). Upon CLC depletion, there was an $\sim 60 \%$ reduction in G-clathrin structures compared with cells treated with control siRNA (Fig. 6a,b,d), and an $\sim 85 \%$ reduction in G-clathrin in cells expressing CLCa-DsRed1 (Fig. 6a,c,d). Though in other respects (that is, labelling of plasma membrane coated pits and trans-Golgi network clathrin), CLCa-DsRed1 appears to be similar to GFP-CLC and has been used for live cell imaging of clathrin ${ }^{35-37}$, here it acts as a dominant negative CLC mutant that specifically affects G-clathrin. Consistent with its effect on G-clathrin, CLCaDsRed1 expression attenuated recycling of the known G-clathrin cargo transferrin receptor and it reduced $\beta 1$-integrin recycling, as 
a

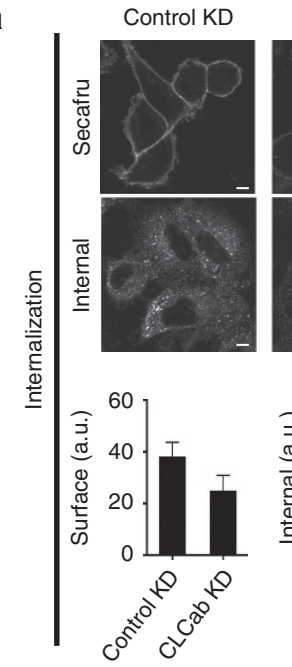

b

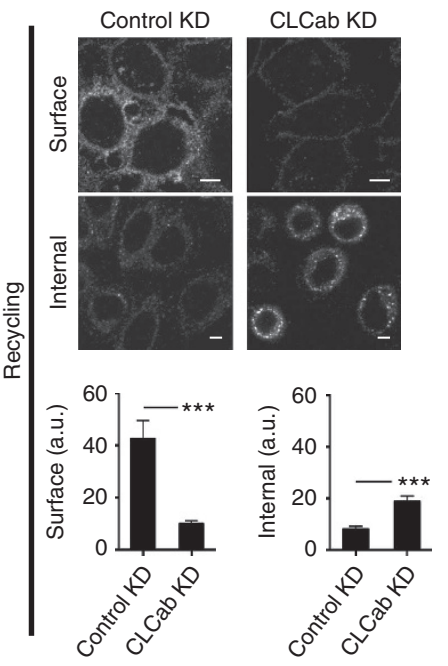

C

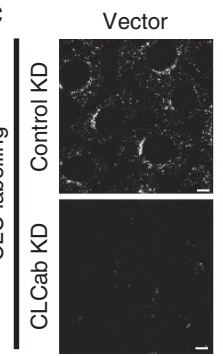

CLCab KD
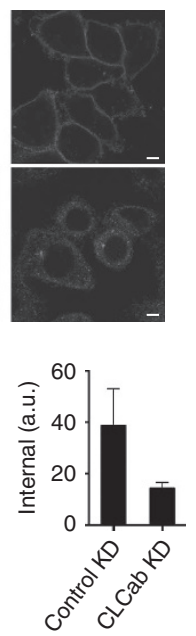

CLCab KD
CLCb WT

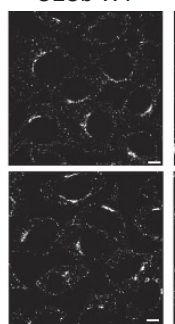

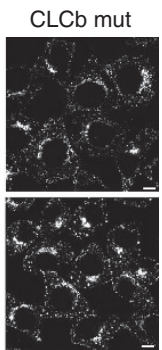

e

d
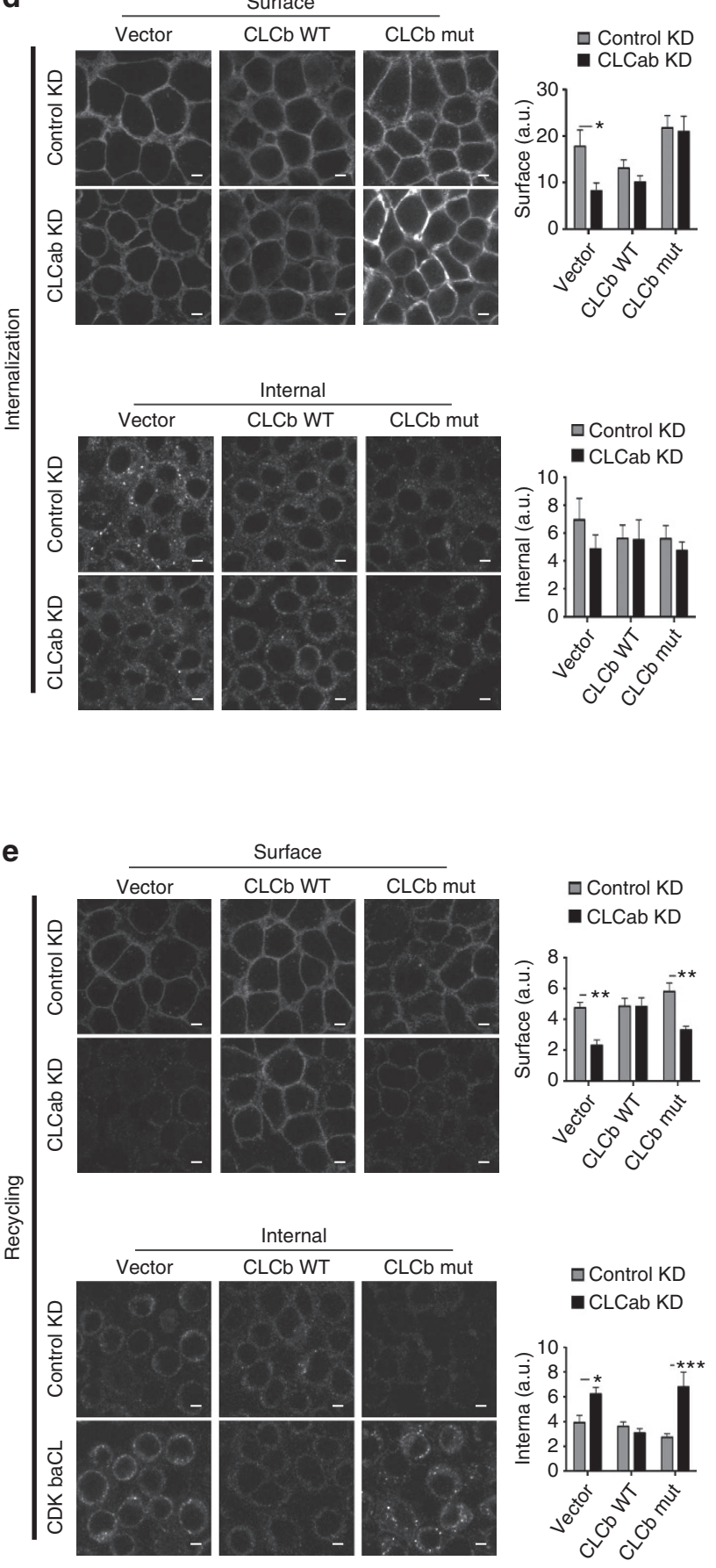

Figure 5 | CLC depletion decreases recycling of inactive $\boldsymbol{\beta 1}$-integrin. (a) siRNA-treated HeLa cells were allowed to internalize antibody specific for inactive $\beta 1$-integrin for $30 \mathrm{~min}$ at $37^{\circ} \mathrm{C}$. Cells were fixed and stained with Alexafluor-conjugated secondary antibody to visualize surface integrins. For analysis of internal integrins, surface antibody was stripped by acid wash before fixation, followed by permeabilization. Quantification of surface (bottom left) and internal (bottom right) fluorescent labelling of inactive $\beta 1$-integrin antibody following internalization, which are presented as raw mean fluorescent intensities (mean \pm s.e.m.; $n=3 ; P=$ not significant, Student's $t$-test). (b) Cells were treated as in $\mathbf{a}$, followed by surface antibody stripping by acid washing after the $30 \mathrm{~min}$ internalization period. Cells were then placed at $37^{\circ} \mathrm{C}$ for $30 \mathrm{~min}$ to chase integrins back to the cell surface. Cells were fixed and processed as in a. Quantification of surface (bottom left) and internal (bottom right) fluorescent labelling of inactive $\beta 1$-integrin antibody following recycling (mean \pm s.e.m.; $n=3$; ${ }^{\star \star \star} P<0.005$, Student's t-test). (c) HeLa clones expressing the vector, siRNA-resistant WT CLCb or siRNA-resistant mutant (mut) $\mathrm{CLCb}$ were treated with control or CLCab-targeting siRNA and immunostained for CLC. (d) Internalization of inactive $\beta 1$-integrin antibody was assessed as in $\mathbf{a}$ in siRNA-treated HeLa clones that expressed the vector, siRNA-resistant WT CLCb or siRNA-resistant mutant CLCb. Quantification of surface (top right) and internal (bottom right) fluorescent labelling of inactive $\beta 1$-integrin antibody following internalization (mean \pm s.e.m.; $n=3$; ${ }^{\star} P<0.05$, two-way analysis of variance (ANOVA) followed by Bonferroni post hoc test). (e) Recycling of inactive $\beta 1$-integrin antibody was assessed as in $\mathbf{b}$ in siRNA-treated HeLa clones that stably expressed the vector, siRNA-resistant WT CLCb or siRNA-resistant CLCb. Quantification of surface (top right) and internal (bottom right) fluorescent labelling of inactive $\beta 1$-integrin antibody following recycling (mean \pm s.e.m.; $n=3 ;{ }^{\star} P<0.05,{ }^{\star \star} P<0.01,{ }^{\star \star \star} P<0.005$, two-way ANOVA followed by Bonferroni post hoc test). Scale bars, $7.5 \mu \mathrm{m}$ for all panels. a.u., arbitrary unit. 
assessed by the biotinylation-based recycling assay (Fig. 6e,f and Supplementary Fig. 5a). Linking this G-clathrin recycling pathway to migration, expression of CLCa-DsRed1 impaired migration of H1299 cells towards a gradient of EGF, visualized by tracking transiently transfected fluorescent cells in a Dunn chamber assay (Fig. $6 \mathrm{~g}$ and Supplementary Fig. 5b). The effect of CLCa-DsRed1 expression was similar to the effect of CLC depletion in the same Dunn chamber assay, where transfected cells were visualized by co-transfection of mCherry and siRNA (Fig. 6h and Supplementary Fig. 5c). HeLa cells expressing CLCaDsRed1 or depletion of CLCs demonstrated a reduction in speed as well as displacement, compared with control cells (Fig. 6i-l). This migration behaviour on matrigel in the Dunn chamber contrasted with the marginal reduction of speed observed in the wound-healing assay upon CLC depletion (Figs 2 and 3). Matrigel contains ligands for several integrins including $\beta 1$-integrin,
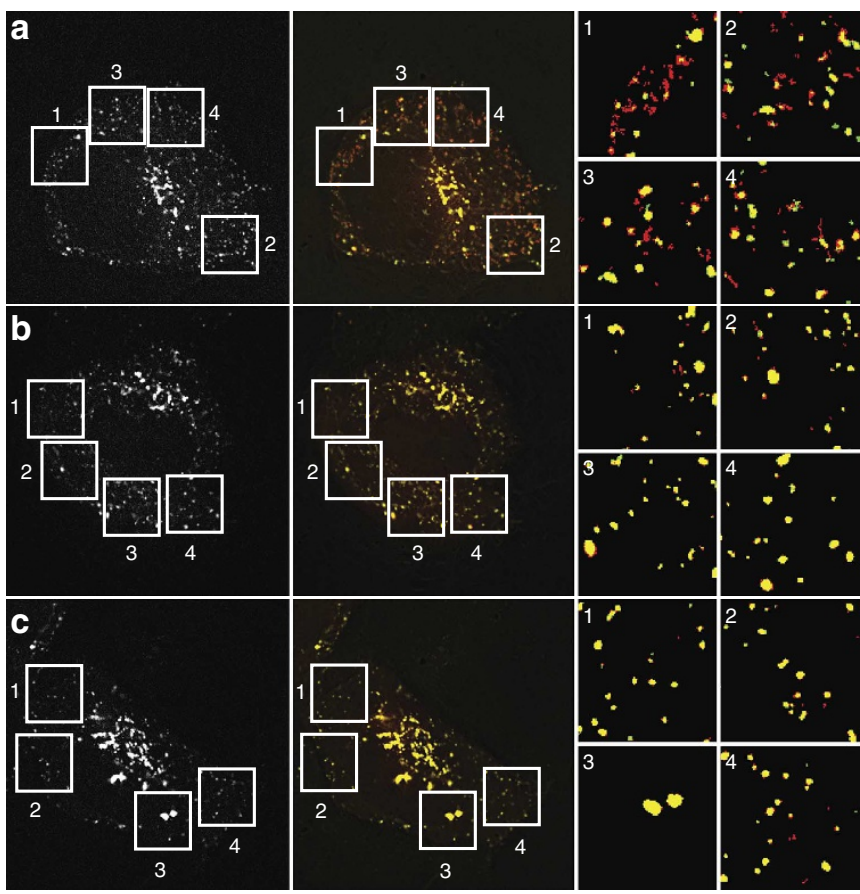
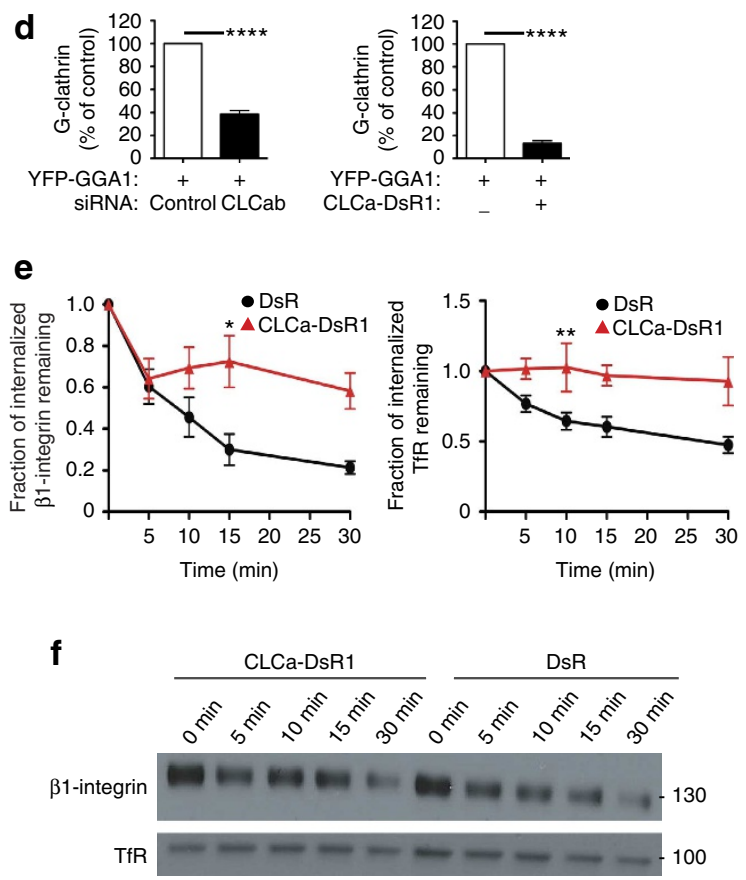
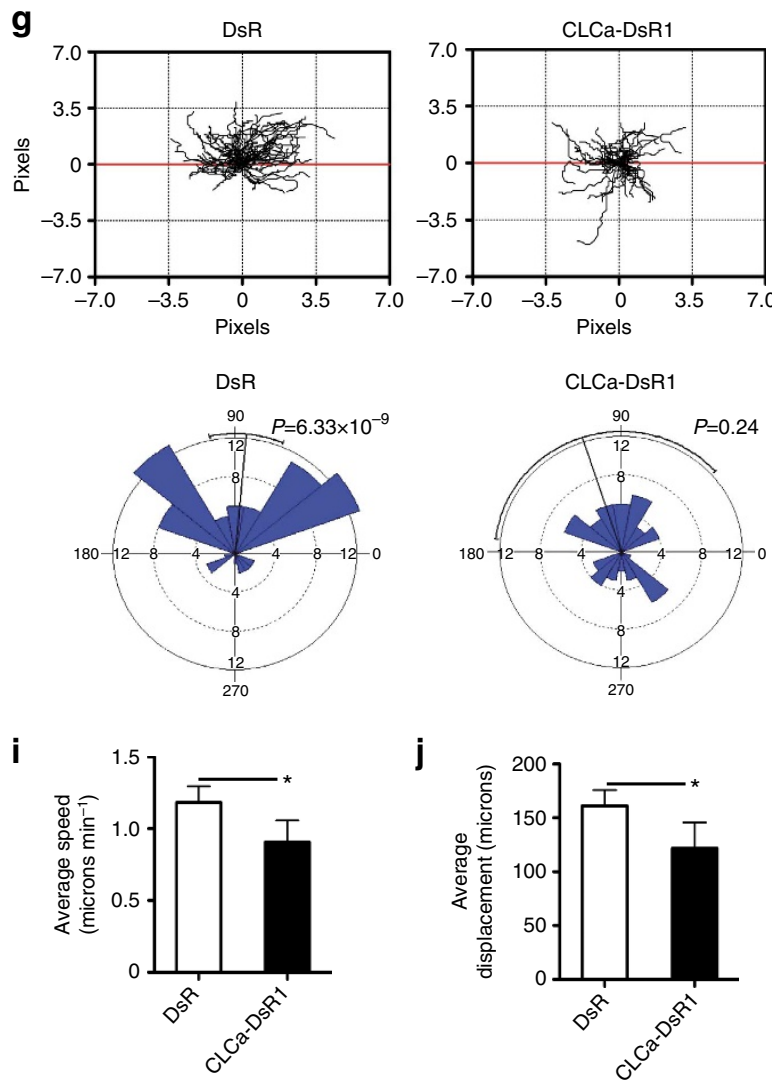

h
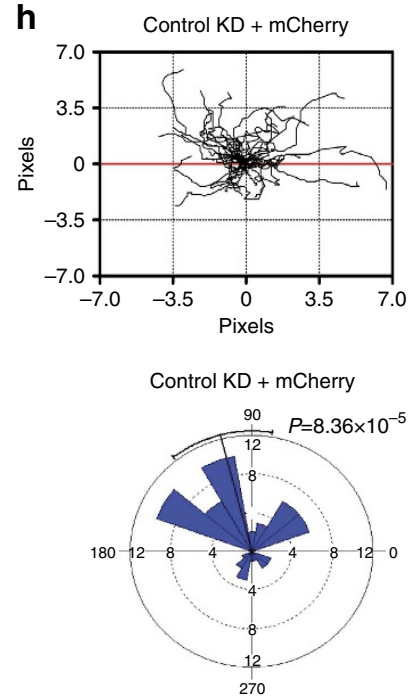

k
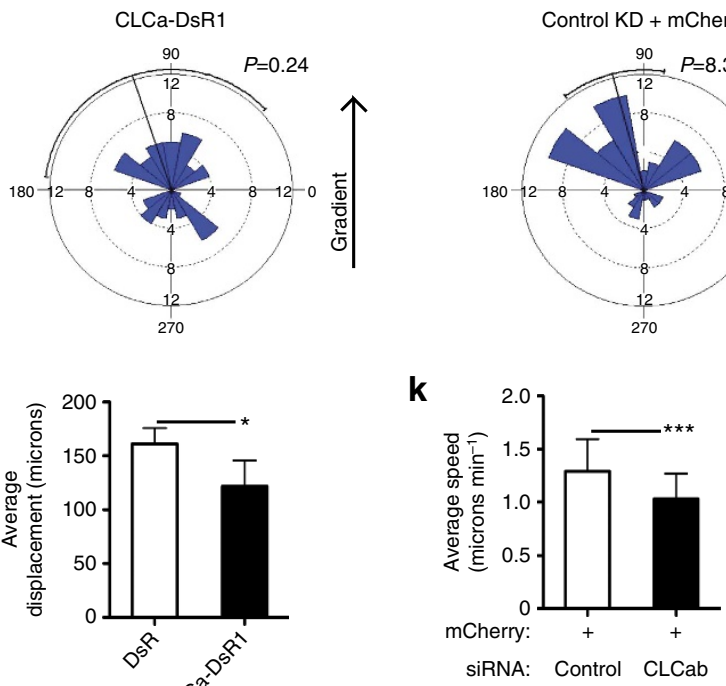

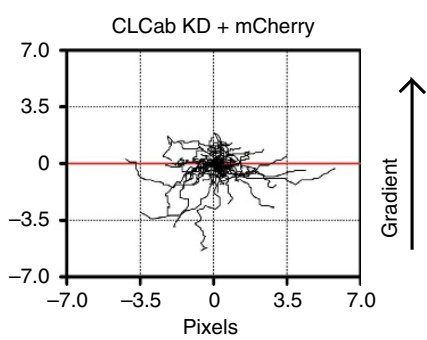

CLCab KD + mCherry
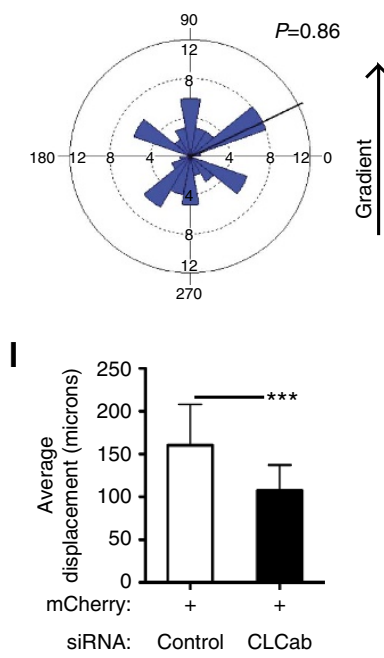
therefore migration on this substrate could be more sensitive to an integrin recycling defect. Together these results implicate CLC as an active component of the G-clathrin rapid recycling pathway for $\beta 1$-integrin as well as transferrin receptor, and demonstrate the importance of this pathway in cell migration.

CLCb expression increases in migratory trophoblast cells. Villi of the placenta are covered by villous trophoblast (VT) cells of fetal origin. Specialized anchoring villi are formed where the placenta contacts the uterine wall from where migratory extravillous trophoblast (EVT) cells invade the uterine lining. This infiltration results in remodelling of maternal arteries, leading to placental vascularization $^{38}$. In search of a physiological correlate for the role of CLC in migration, we queried available data from a microarray analysis comparing gene expression in VT versus EVT ${ }^{39}$ cells. As expected, several genes involved in cell adhesion and migration were upregulated in the migratory EVT cells compared with non-migratory VT cells (Fig. 7a). Strikingly, we noted that non-neuronal $\mathrm{CLCb}$ was one of the top genes upregulated in invasive EVT cells (Fig. 7a). No such increase in expression was observed for non-neuronal or neuronal CLCa, neuronal CLCb or CHC17 transcripts in these cells, suggesting that CLCb may uniquely contribute to the migratory potential of EVT cells.

To establish whether CLCb protein increases in EVT cells as a result of increased gene expression, we analysed the distribution of clathrin and CLCs in placental tissue sections by tracking the level of immunofluorescence across tissue sections from VT to EVT cells. These trophoblast regions were identified by immunolabelling for standard markers of all trophoblast (cytokeratin) and of EVT cells, which express HLA-G on their surface during invasion ${ }^{40}$ (Fig. 7b). Consistent with known distribution, cytokeratin expression was observed in cytotrophoblast cell columns and interstitial trophoblast cells present in maternal tissue. The EVT cells showed expression of HLA-G as expected (Fig. 7b). CLCb was barely detectable in the VT cells (Fig. 7b), but labelling increased in EVT cells (indicated by arrowheads in Fig. $7 \mathrm{~b}$ ) in the vicinity of maternal vessels. In contrast, CLCa and CHC17 expression were uniform across all trophoblast regions (Fig. 7b). These observations were reproduced in other placental sections from an unrelated individual, and agree with the array data. Thus tissue expression analysis suggests a physiological role for $\mathrm{CLCb}$ in trophoblast invasion that is consistent with a role for CLC in cell migration.

\section{Discussion}

This study establishes a role for CLC subunits and their Hip binding in the recycling of inactive $\beta 1$-integrin and in cell migration. Moreover, we show that the rapid endosomal recycling pathway mediated by G-clathrin structures depends on CLC function and that a specific inhibitor of G-clathrin attenuates cell migration and $\beta 1$-integrin recycling, as well as recycling of the known G-clathrin cargo, transferrin receptor. Identification of G-clathrin as a mediator of rapid endosomal recycling during migration and demonstrating its CLC dependence, implicates the CLC-binding Hip proteins as additional actin organizers needed for this pathway, shown previously to also require the actinorganizing proteins Wiskott-Aldrich syndrome protein family homologue (WASH) and actin-related protein (Arp) 2/3 complex $^{41}$. In support of a key role for CLC-dependent G-clathrin in cell migration, we observed upregulation of $\mathrm{CLCb}$ in migrating trophoblast, providing an in vivo correlate for our findings.

The role of clathrin in migration mediated by CLC defined here is distinct from that previously established by depletion of the $\mathrm{CHC17} \mathrm{CHC}$, which revealed a role for clathrin in endocytosis of inactive $\beta 1$-integrin during migration ${ }^{18}$. These distinguishable clathrin-mediated trafficking pathways for inactive $\beta 1$-integrin (Fig. 8) both influence focal adhesions. $\mathrm{CHC} 17$ depletion increased focal adhesions, as expected from reduced integrin uptake, whereas CLC depletion had the opposite effect (Fig. 1), explained by reduced recycling of $\beta 1$-integrin with persistent internalization. Supporting the concept that clathrin functions in balanced membrane traffic pathways during cell migration, overexpression of a CLCb mutant that reduced actinassociated clathrin plaques at the cell-substrate interface was shown to enhance migration ${ }^{23}$. That particular QQN-CLCb mutant studied, is defective for both Hip binding and for regulation of clathrin assembly ${ }^{13,14,42}$, so could not distinguish between CLC and $\mathrm{CHC17}$ roles in migration. Here, using different mutants, we show that both migration and $\beta 1$-integrin recycling depend on the minimal CLC-Hip binding residues, without altering the CLC residues involved in clathrin assembly. The CLC-dependent G-clathrin recycling pathway could potentially influence levels of growth factor receptors and affect directional migration, also explaining variable migration phenotypes seen with different cells and interference protocols. We further note that $\mathrm{CHC17}$ has been implicated in lamellipodium formation, and could affect cell migration via recruitment of the Scar-Wave complex to the leading edge of the cell $^{29}$. Thus clathrin plays multiple roles in cell motility, expanded by the function of CLC and G-clathrin in $\beta 1$-integrin recycling established here.

Defining the roles of CLC in cellular clathrin function has been challenging, although in vitro biochemical studies have demonstrated a role for CLC in regulating both clathrin assembly and Hip interactions with $\operatorname{actin}^{3,16}$. The requirement for CLCs in

Figure 6 | Expression of CLCa-DsRed1 or CLC depletion causes loss of G-clathrin structures and CLCa-DsRed1 reduces recycling and cell migration. (a-c) HeLa-M cells transfected with YFP-GGA1, and with either (a) control siRNA, or (b) CLCab siRNA or with (c) CLCa-DsRed1 were imaged using continuous $30 \mathrm{~ms}$ exposures ( $1 \mathrm{~s}$ total). A single image from each sequence is shown on the left. Combined maximum projections (red), which reveal both dynamic and stationary GGA1 structures and sum projections (green), which report stationary structures, are shown at the right, with overlap in yellow ( $54 \mu \mathrm{m}$ square panels). Dynamic G-clathrin appears red. Boxed areas are magnified (right) and pixel intensities binarized for clarity. (d) Percent of G-clathrin relative to controls in HeLa-M cells transfected with CLCab siRNA or CLCa-DsRed1 (CLCa-DsR1) in a-c. G-clathrin for each condition was calculated as the difference between the maximum projection and sum projection of YFP-GGA1 signals (see Methods for details) (mean \pm s.e.m.; $n=4$ for CLCab, knockdown (KD) $n=2$ for CLCa-DsR1, ${ }^{\star \star \star \star} P<0.0001$, Student's unpaired $t$-test). (e) Recycling of $\beta 1$-integrin (left) and TfR (right), assessed by biotinylation as in Fig. 4d, in DsRed1 (DsR)- or CLCa-DsR1-transfected HeLa cells (mean \pm s.e.m.; $n=7 ;{ }^{\star} P<0.05$, ${ }^{\star \star} P<0.01$, two-way analysis of variance followed by Bonferroni post hoc test). (f) Representative immunoblots from one recycling experiment in e. Lysate samples are shown in Supplementary Fig. 5a. (g,h) Dunn chamber migration tracks of H1299 cells transfected with (g) DsR or CLCa-DsR1 or (h) mCherry plus control or CLCab siRNA in the presence of an EGF gradient (source at $90^{\circ}$ ). Tracks in the absence of a gradient are shown in Supplementary Fig. 5 b,c. All tracks were set to a common origin (intersection of $x$ [red] and $y$ axes). Circular histograms depict proportion of cells whose final position lies within each of $1820^{\circ}$ sectors (mean and 95\% confidence interval indicated by black line; at least 50 cells analysed from four independent experiments for each condition, $P$-values, Raleigh uniformity test). (i) Average speed and (j) displacement of DsR- and CLCa-DsR1-transfected gradient-exposed cells. (k) Average speed and (I) displacement of control-treated and CLCab-depleted gradient-exposed cells. (i-I: mean \pm s.e.m.; $n=4{ }^{\star} P<0.05$, ${ }^{\star \star} P<0.01$, Student's paired $t$-test). 
a

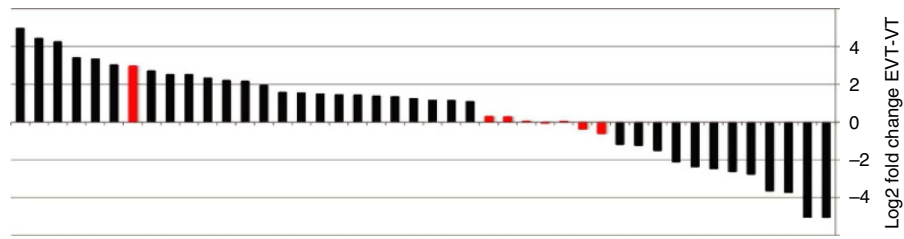

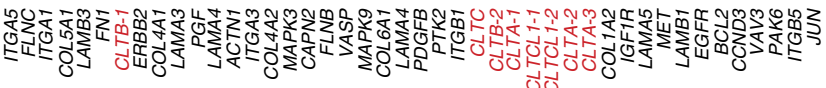

b

Overlay
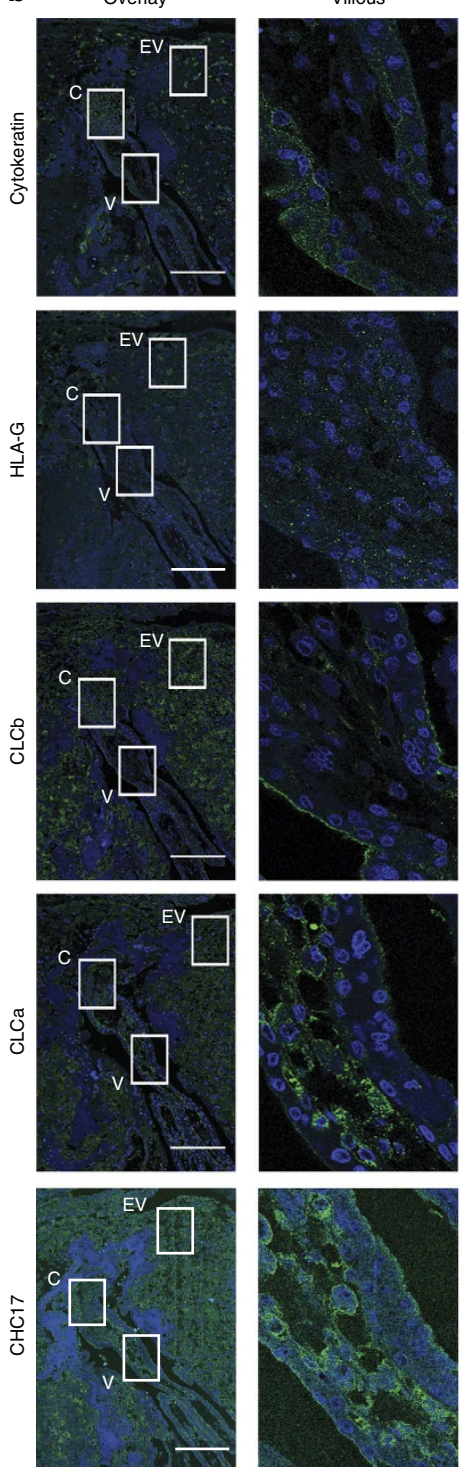

Columnar
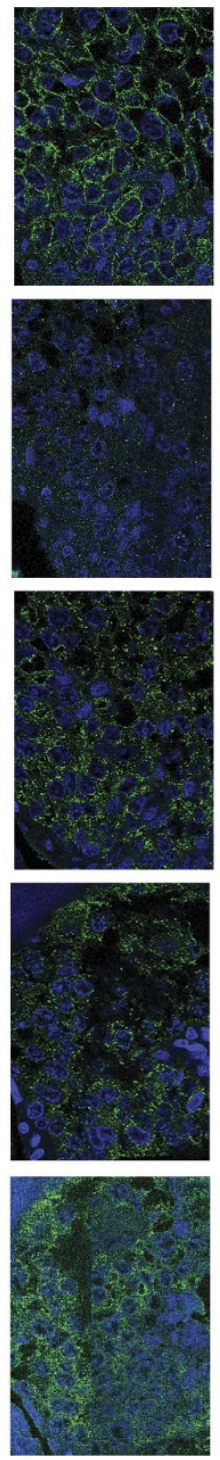

Extravillous
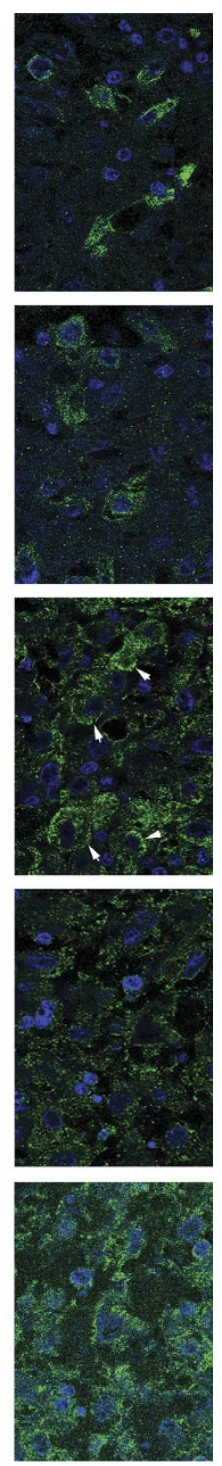
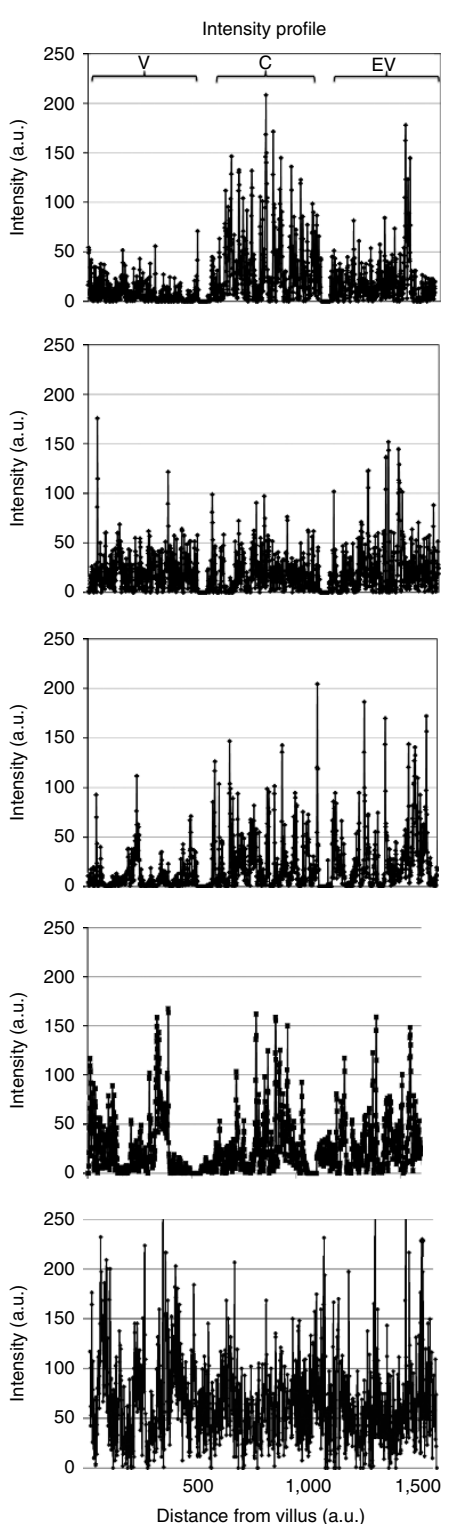

Figure 7 | CLCb is upregulated in invasive extravillous trophoblast cells. (a) Microarray analysis of mRNA in human trophoblast cells showing relative gene expression levels in extravillous trophoblast (EVT) cells compared with villous trophoblast (VT) cells. Genes encoding clathrin subunits and their transcript levels are indicated in red. CLTB encodes CLCb, CLTA encodes CLCa, CLTC encodes CHC17 and CLTCL1 encodes CHC22. For three genes encoding clathrin subunits, more than one probe was used to detect splice variants and these are numbered. Probes CLTB-1, CTLA-2, CLTA-3 and CLTCL1-1 detect all variants, CLTB-2 and CLTA-1 detect the neuronal splice variants of CLCs and CLTCL1-2 detects a second splice variant encoded by this gene. (b) Serial sections of normal, non-cancerous human placental tissue were stained for CLCb, CLCa, CHC17 and trophoblast markers, as indicated. Cytokeratin staining depicts cytotrophoblast cell columns (columnar) and invading extravillous trophoblast cells developing from placental villi. Extravillous trophoblast was distinguished from villous trophoblast on the basis of HLA-G expression. Arrowheads depict extravillous trophoblast cells with high CLCb expression. Intensities of the staining are quantified as arbitrary units (a.u.) and illustrated on the right as line profiles across each box shown at the far left. $\mathrm{V}$, villous; C, columnar; EV, extravillous trophoblast. Scale bars, $500 \mu \mathrm{m}$. 

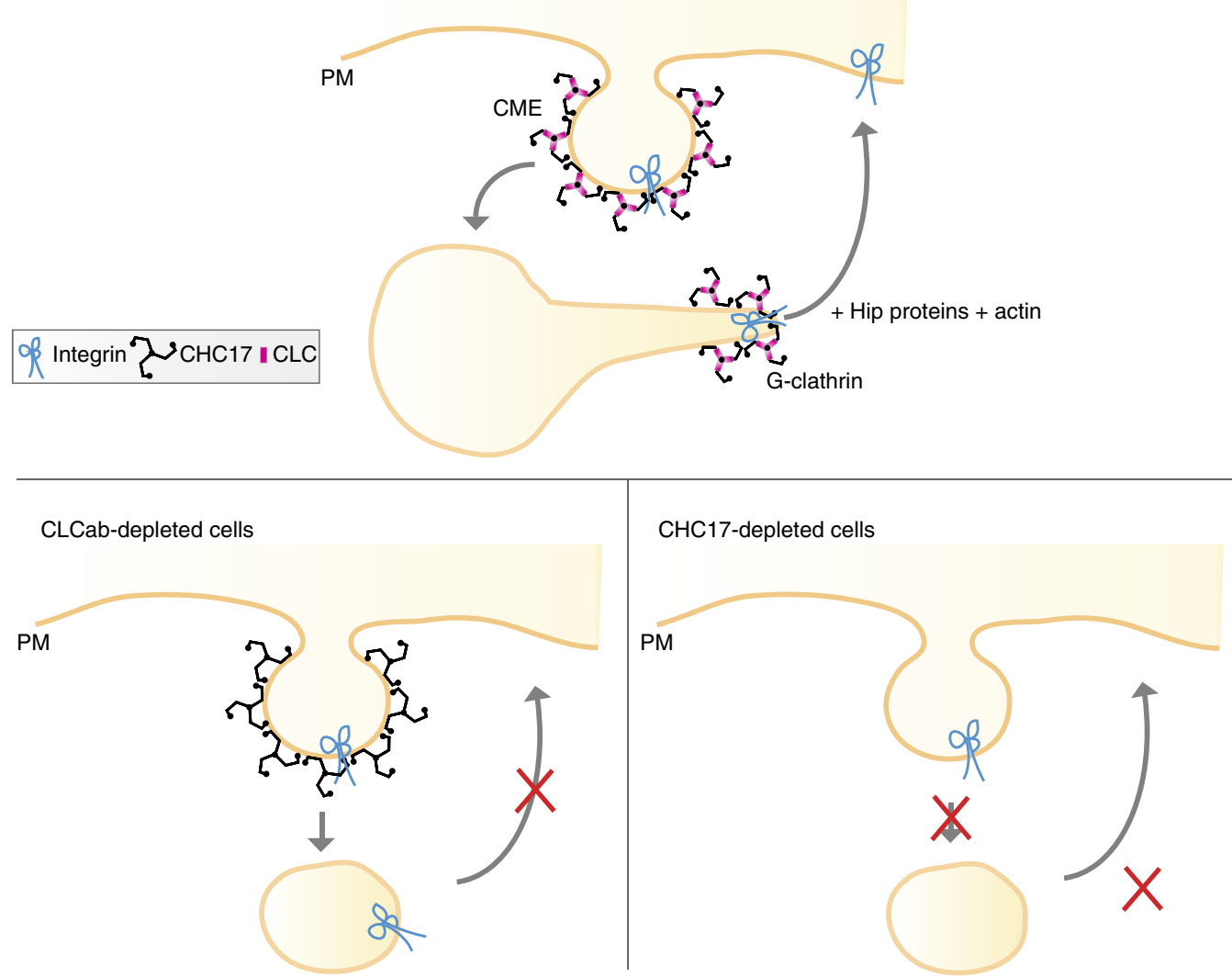

Figure 8 | Model for the roles of clathrin in inactive $\beta 1$-integrin membrane traffic during cell migration. Clathrin is involved in both endocytosis from the plasma membrane (PM) and recycling of inactive $\beta 1$-integrin from endosomes. CLC is required for the recycling of $\beta 1$-integrin back to the cell surface via G-clathrin structures, but not needed for clathrin-mediated endocytosis (CME). Loss of the total clathrin by $\mathrm{CHC17}$ depletion inhibits all pathways, but manifests itself as an endocytosis defect. We note that non-clathrin-mediated endocytic routes can also contribute to integrin uptake ${ }^{21}$.

CME is limited to some G-protein-coupled receptors, but dispensable for many common CME cargo ${ }^{5-7}$. On the other hand, uptake of cargo from membranes under tension and of large pathogen particles depend on CLC interactions with Hip proteins $^{8-10,43}$. In these latter situations, clathrin serves an actin organizer, a role that it also plays at certain bacterial-host interfaces 8 and during adherens junction formation ${ }^{44}$. Hip1R has been implicated in actin-based movements of Rickettsia and clathrin is required for actin polymerization during vaccinia infection ${ }^{45,46}$, suggesting that CLC may be similarly involved. Here we add to the repertoire of actin-based functions mediated by CLC. Upon CLC depletion, we observe disorganized, branched actin filaments at the cell periphery and reversing the depletion phenotype requires CLC-Hip interaction. We further show that CLC is needed for G-clathrin, as well as migration and recycling, which also depend on CLC-Hip binding. G-clathrin has a characteristic 'gyrating' behaviour in the cell periphery and represents endosome-associated clathrin-coated tubules that mediate rapid recycling ${ }^{26}$. We propose that CLC is needed for G-clathrin to function in recycling and migration because, through Hip binding, CLC could connect tubule-associated clathrin coats with actin for directional extension from endosomes. Clathrin-coated carriers bound to ARF1 and adaptor protein 1 (AP1) in the trans-Golgi network form actinbased tubules ${ }^{47}$, and G-clathrin is also nucleated by ARF1 and ARF6 (ref. 34). CLC depletion was reported to change actin morphology in a perinuclear compartment labelled for the AP1 adaptor ${ }^{6}$, consistent with our finding a function for CLC on endosome-associated clathrin and possibly a further role for CLC on trans-Golgi network tubule clathrin. It is notable that
CLCa-DsRed1 acts as a dominant negative mutant in the G-clathrin recycling pathway, though it has been used as a marker for live cell imaging of clathrin and functions normally at the plasma membrane. This is likely a consequence of a unique intolerance of G-clathrin to oligomerization of CLCs, driven by the obligate tetrameric quaternary structure of DsRed1 (ref. 48). In contrast, either $\mathrm{N}$ - or C-terminal-tagged fusions of CLCs with predominantly monomeric GFP, YFP or mCherry proteins all give robust evidence of G-clathrin.

The observation that CLCb is markedly upregulated in invasive trophoblast further strengthens our assignment of an important role for CLC in migration. In our cellular studies, there was no distinction between CLCa and CLCb function in migration or influence on actin organization or recycling, as each CLC isoform seems to substitute for the other in restoring the knockdown phenotypes observed here. Tissue analysis has indicated that the CLCa:CLCb ratio is characteristic of different tissues and that CLCa is usually the more abundant ${ }^{49,50}$. Indeed, the tissue section staining we performed shows ubiquitous expression of CLCa in all placental regions. Currently little is known about clathrin gene regulation, but it may be that $\mathrm{CLCb}$ expression is more subject to transcriptional regulation, allowing its increase during differentiation. Alternatively or additionally, CLCa and CLCb share only $60 \%$ sequence identity outside of their identical Hip-binding regions, so it is possible that there are functional differences in flanking sequences that affect Hip-actin interactions required for trophoblast migration, again needing further biochemical evaluation.

Pre-eclampsia occurs when trophoblast invasion of the uterine wall is defective ${ }^{40}$, so CLC expression might be a 
therapeutic target for women with pregnancy disorders. Another physiological correlate, not addressed directly here, is the observation that Hipl expression is upregulated in metastatic breast and prostate cancers as well as other malignancies ${ }^{51-53}$. It is conceivable that increased Hip expression enhances migratory behaviour. Migration of H1299 cells was more sensitive to CLC and Hip knockdown than migration of the slower-moving HeLa cells, which express less Hip1 protein. Thus, defining the role of CLC in cell migration establishes a potential mechanism for metastasis of cancers with increased Hip expression that might be considered as a molecular target for anti-metastatic strategies.

\section{Methods}

siRNAs and plasmids. siRNA duplexes were synthesized by Qiagen. Targeting sequences were published as follows, CHC17, CLCa and $\mathrm{CLCb}^{5}$, CHC22 (ref. 31), or designed by Qiagen for Hip proteins (5'-CAGGAACTTGCCACAAGCCAA-3', Hip1 and 5'-CTCCGACATGCTGTACTTCAA-3, Hip1R). The CLC siRNAresistant construct (WT, with a silent mutation at serine 88 from AGT to TCA) or CLC-Hip-binding resistant mutant (I38A and D25A) of bovine brain CLCa was generated from a $5^{\prime}$-HA-tagged pcDNA3-based construct ${ }^{13}$, with QuikChange site-directed mutagenesis kit (Stratagene). Mutant human CLCa (I43A) and CLCb (I35A) encoding constructs that are siRNA resistant were similarly produced without the HA tag.

Tissue culture and transfections. HeLa cells (lab stock) and the H1299 lung cancer cell line (gift of J.M. Bishop, UCSF) were cultured in DMEM and RPMI1640 , respectively, supplemented with 10\% fetal bovine serum (FBS) (Hyclone) and antibiotics. Cells were trypsinized and transfected at $\sim 30 \%$ confluency with siRNA using the HiPerfect reagent (Qiagen), following the manufacturer's protocols. For transient rescue experiments, cells were transfected with plasmid DNA one day after siRNA transfection, using Lipofectamine 2000 (Life Technologies) according to the manufacturer's instructions. Cells were analysed $72 \mathrm{~h}$ post siRNA treatments.

HeLa and H1299 clones expressing the empty vector, siRNA-resistant human WT CLCa or CLCb, or human Hip-binding resistant mutant CLCa (I43A) or CLCb (I35A) were established by transfection of plasmids into HeLa and H1299 cells using Lipofectamine 2000 (Life Technologies) as above. G418 (Life Technologies) was added to the cell culture medium at $500 \mu \mathrm{g} \mathrm{ml}^{-1} 48 \mathrm{~h}$ post transfection to select clones. Individual G418 resistant colonies were selected and expanded into cell lines 3 weeks later, followed by confirmation of plasmid expression by immunoblotting.

Antibodies and other reagents. The following monoclonal antibodies were used for immunofluorescence: anti-CHC17 $\left(2 \mu \mathrm{g} \mathrm{ml}^{-1}\right.$; X22 (ref. 54)), cortactin (1:500; Millipore, number 05-180), paxillin (1:500; Millipore, number 05-417), anti-HA (1:100; Covance, number MMS-101P). Rat anti-CD29 mAb13 against inactive $\beta 1$-integrin $\left(5 \mu \mathrm{g} \mathrm{ml}^{-1}\right.$; BD biosciences, number BDB552828), rabbit polyclonal antisera against the conserved region of CLCs ${ }^{55}$, and Hip1R (1:100; Millipore, number AB9882) were also used. Secondary labelling was done with Alexafluor488 (1:500; Life Technologies, number A-11001) or 555-conjugated secondary antibodies (1:500; Life Technologies, number A-21422). Alexafluor 647-phalloidin (1:100; Life Technologies, number A-22287) was used to stain F-actin. The following antibodies were used for immunoblotting: anti-CHC17 (1:1,000; TD.1 (ref. 56)), Hip1R (1:500; Millipore, number AB9882), $\beta 1$-integrin (1:1,000; BD Biosciences, number 610467), TfR (1:1,000; BD Biosciences, number 612124), HA (1:1,000; Covance, number MMS-101P), Hip1 (1:500; Sigma, number HPA013606), anti- $\beta$-actin (1:2,000; Sigma, number A5441) and $\alpha$-tubulin (1:5,000; Sigma, number T6199).

Immunoblotting. Cells were lysed in a buffer containing $50 \mathrm{mM}$ Tris (pH 7.2), $1 \%$

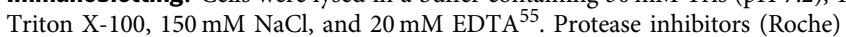
were added to the buffer before lysis. Protein content was determined using Bradford reagent (Bio-Rad). Equal amounts of cell lysates were resolved on pre-cast SDS-PAGE gels (Invitrogen) and transferred onto nitrocellulose membranes (Bio-Rad), followed labelling with primary and secondary antibodies.

\footnotetext{
Migration assays. HeLa or H1299 cells treated with $20 \mathrm{nM}$ siRNA for $48 \mathrm{~h}$ were plated at $2 \times 10^{5}$ cells ml $^{-1}$ in culture inserts (Ibidi GmbH) affixed to wells in glass-bottom (MatTek) or tissue culture plastic (BD Falcon) 24-well plates and allowed to adhere for $24 \mathrm{~h}$. Before imaging, culture inserts were removed to create the wound, and the cells were washed in culture medium with $1 \%$ FBS. Migration across the wound was imaged once every $10 \mathrm{~min}$ for 24 (HeLa) or 10-15 h (H1299) using a $\times 10$ objective on a Leica TCS SP5 microscope. Cells were maintained at $37^{\circ} \mathrm{C}$ and $5 \% \mathrm{CO}_{2}$ for the duration of the experiment. Cells were tracked using the MTrackJ plugin of ImageJ. Twenty cells were tracked per condition for each experiment. Wound-healing assays for parent HeLa and H1299 cell lines were
}

performed on glass, whereas migration of CLC transfectants was assessed on plastic, which better supported migration.

Chemotaxis assays were performed using the Dunn chamber (Hawksley DCC100). Two thousand four hundred H1299 cells transfected with either DsRed1 or CLCa-DsRed1, or mCherry and either control or CLCab siRNA, were plated on $2 \%$ matrigel-coated coverslips and allowed to adhere for $6 \mathrm{~h}$ in complete medium. Cells were then starved overnight in a medium containing $0.01 \%$ serum. The coverslip was placed onto the Dunn chamber in which both inner and outer wells were filled with control medium (medium containing $0.01 \%$ serum) and three sides were sealed with wax. Media from the outer well was drained using filter paper and control media containing $1 \mathrm{ng} \mathrm{ml}^{-1}$ EGF was inserted into the outer well and the fourth side was then sealed with wax. To assess migration in the absence of a gradient, all four sides of the coverslip were sealed with wax once the coverslip was placed onto the chamber containing control medium. The Dunn chamber was then placed on a heated stage at $37^{\circ} \mathrm{C}$. Time-lapse images were taken every $5 \mathrm{~min}$ for $3 \mathrm{~h}$ using a $\times 10$ objective on a Leica TCS SP5 microscope. Migration of non-dividing DsRed1-, CLCa-DsRed1- or mCherry-expressing cells were tracked using the MtrackJ plugin of ImageJ.

Immunofluorescence. Cells grown on coverslips were fixed using 4\% PFA (Ted Pella, Inc.) for $10 \mathrm{~min}$. When viewing HA-tagged CLCa with other proteins stained by a monoclonal antibody, Alexafluor 594-conjugated monoclonal antibody to HA (1:500; Covance, number A594-101L) was applied after incubation with secondary antibody. Images were collected with an API DeltaVision DV3 restoration or a Leica TCS SP5 confocal microscope. To determine the percent of cell periphery occupied by focal adhesions, the sum of edges stained for paxillin along the cell perimeter was divided by the cell perimeter, with both measured using ImageJ.

Paraffin-embedded serial placental sections from a de-identified normal human sample were deparaffinized in xylene and rehydrated in reducing concentrations of ethanol (100-70\%). Following washes with water and PBS, antigen epitopes were unmasked by boiling slides in $10 \mathrm{mM}$ sodium citrate for $10 \mathrm{~min}$. Cooled slides were washed in PBS, and sections were permeabilized in blocking buffer (PBS, 5\% BSA and $2 \%$ goat serum) with $0.1 \%$ Triton X-100 for $30 \mathrm{~min}$. Sections were incubated in the following primary antibodies diluted in the blocking buffer for $1 \mathrm{~h}$ at room temperature: $5 \mu \mathrm{g} \mathrm{ml}^{-1}$ of LCB.1 (for CLCb) ${ }^{57}$, of X16 (for CLCa) ${ }^{54}$ and of X22 (for $\mathrm{CHC17}^{54}, 10 \mu \mathrm{g} \mathrm{ml}^{-1}$ of anti-HLA-G (MEM-G/1, SantaCruz, number sc-51674), or $0.2 \mu \mathrm{g} \mathrm{ml}^{-1}$ of anti-cytokeratin (Clone MNF116, Dako Cytochromation, number M082101-2). Following washes in PBS with 0.1\% Tween-20, secondary antibodies $\left(10 \mu \mathrm{g} \mathrm{ml}^{-1}\right)$ and TO-PRO (Life Technologies, number T3605) were incubated with $10 \%$ human $\mathrm{AB}$ serum for $30 \mathrm{~min}$. After $30 \mathrm{~min}$ room temperature incubation, the slides were washed in PBS with $0.1 \%$ Tween-20 and mounted. Images in Fig. 7 were cropped to depict the same area using Adobe Photoshop CS3. The intensity of all channels was adjusted equally across all images. The difference in protein expression between the villous trophoblast $(\mathrm{V})$, cytotrophoblast column $(\mathrm{C})$ and the extravillous trophoblast (EV) cells within an image was quantified as follows. A line was drawn within the boxed regions in the mosaic image and the plot profile function in Image) was used to calculate pixel intensities along the line. Approval to use these tissues was obtained by the UCSF Committee on Human Research and the Cambridge Local Research Ethics Committee.

MTT assay. To measure cell proliferation, MTT assays (Molecular Probes) were performed according to the manufacturer's protocol. Briefly, 10,000 cells were plated in triplicate in each well of a 96-well plate. After 24 or $48 \mathrm{~h}$, fresh media was added to the cells followed by $12 \mathrm{mM}$ MTT reagent for $4 \mathrm{~h}$ at $37^{\circ} \mathrm{C}$. After the incubation period, dimethylsulphoxide was added to the cells to dissolve the formazan and absorbance was read at $540 \mathrm{nM}$.

Expression profiling by microarray. RNA expression levels in primary trophoblast cells were determined as previously described ${ }^{39}$. Briefly, biotinylated cRNA was synthesized from total RNA isolated from primary trophoblast cells using the Illumina RNA amplifcation kit (Ambion). Labelled cRNA was hybridized to Illumina Human $\mathrm{HT}_{-12} \mathrm{~V}_{3}$ BeadArrays according to the manufacturer's protocol. Data were processed by converting signal intensities to $\log _{2}$ expression units. Differential gene expression between two groups of samples was determined using the output of a moderated $t$-test. $P$-values were converted to corrected $q$-values using the previously published FDR method ${ }^{58}$ and probe-sets with $q<0.01$ (FDR $1 \%$ ) were regarded as having significant differential expression between groups.

Antibody-based internalization and recycling assays. Cells plated on glass coverslips were treated with siRNA for $72 \mathrm{~h}$, then serum starved for $1 \mathrm{~h}$, followed by labelling with $5 \mu \mathrm{g} \mathrm{ml}^{-1}$ antibody to inactive (mAb13) $\beta 1$-integrin on ice for $15 \mathrm{~min}$. Cells were washed to remove excess antibody and incubated in prewarmed culture medium supplemented with $10 \% \mathrm{FBS}$ at $37^{\circ} \mathrm{C}$ for $30 \mathrm{~min}$ to start integrin internalization. To determine surface levels of integrins, cells were placed on ice, washed with ice-cold PBS, fixed in ice-cold $4 \%$ PFA, then blocked with $0.2 \%$ fetal goat serum and incubated with Alexafluor-555 secondary antibody for $1 \mathrm{~h}$. To determine internal levels, surface integrins were stripped with ice-cold PBS (pH 2.5) before fixation, followed by permeabilization with $0.2 \%$ Triton X-100. The cells were blocked and incubated with Alexafluor-555 secondary antibody as above. To 
measure recycling, labelled integrins were allowed to internalize at $37^{\circ} \mathrm{C}$ as above, followed by stripping of surface integrins with ice-cold PBS ( $\mathrm{pH}$ 2.5). Cells were washed with ice-cold PBS, then incubated in pre-warmed medium (10\% FBS) for $30 \mathrm{~min}$ at $37^{\circ} \mathrm{C}$ to chase internal integrins to the surface. Surface and internal integrins were determined as above. Images sections were acquired every $0.5 \mu \mathrm{m}$ in a $2.5-\mu \mathrm{m}$ slice through the middle of the cell and merged into maximum projections. The mean fluorescent intensity for each image was obtained by measuring total fluorescence, followed by mean background subtraction using ImageJ. This value was then divided by the total number of cells in each image.

Surface biotinylation and biotinylation-based recycling assays. siRNA-treated cells were washed twice in ice-cold PBS $(\mathrm{pH} 8)$ and surface proteins were biotinylated with $0.5 \mathrm{mg} \mathrm{ml}^{-1}$ Sulfo-NHS-SS Biotin (Thermo Fisher Scientific) in ice-cold PBS (pH 8) for $30 \mathrm{~min}$ at $4{ }^{\circ} \mathrm{C}$. Excess biotin was quenched by washing cells twice with ice-cold $5 \mathrm{mM}$ Tris (pH 7.4), followed by two washes with ice-cold PBS ( $\mathrm{pH}$ 8) and one wash with PBS (pH 7.4). Cells were lysed in PBS with $1 \%$ NP-40 containing protease inhibitors (Roche). Protein concentrations in the samples were determined using Bradford reagent and equal amounts of total protein were loaded onto streptavidin beads (Thermo Fisher Scientific) and incubated at $4{ }^{\circ} \mathrm{C}$ for $1 \mathrm{~h}$. After washing the beads, proteins were eluted by boiling the beads in SDS sample buffer, followed by immunoblotting. Blots were quantified using ImageJ, and the level of surface receptors were normalized to the $\alpha$-tubulin signal from each sample.

For biotinylation-based recycling assays, serum starved siRNA-treated cells were labelled with biotin and the excess washed as above. The cells were then placed at $37^{\circ} \mathrm{C}$ in media containing $10 \% \mathrm{FBS}$ for $30 \mathrm{~min}$ to allow internalization of surface proteins. Surface biotin was then reduced with two $10 \mathrm{~min}$ washes with $50 \mathrm{mM}$ MesNa ( $\mathrm{pH}$ 8.6) in TBS, followed by one $10 \mathrm{~min}$ wash with $20 \mathrm{mM}$ iodoacetamide in TBS, all at $4^{\circ} \mathrm{C}$. Cells were then rinsed with ice-cold PBS and placed at $37^{\circ} \mathrm{C}$ in media containing $10 \%$ FBS for indicated time points to chase internalized proteins to the cell surface. Surface biotin was then reduced again as described, and the cells were lysed, equal amounts of protein loaded onto streptavidin beads, eluted and blotted as mentioned above. The fraction of internalized receptor remaining for each protein was determined from the signal intensity of internalized protein at each time point relative to control cells that had not been placed at $37^{\circ} \mathrm{C}$ after the first surface reduction.

Quantification of G-clathrin by GGA1 movement. Hela-M cells were transfected with either control or CLC-targeting siRNA, and then co-transfected with plasmid encoding YFP-GGA1 $48 \mathrm{~h}$ later, or they were transfected with plasmid encoding either YFP-GGA1 alone or YFP-GGA1 and CLCa-DsRed1. The cells were then imaged $24 \mathrm{~h}$ post DNA transfection by spinning disk confocal microscopy using streaming $30 \mathrm{~ms}$ exposures to collect 30 frame image stacks as previously described ${ }^{34}$. For quantification of G-GGA1, we used a modified assay on the basis of movement area ${ }^{26}$. Briefly, stacks were background corrected using a rolling ball filter $(r=5)$ in ImageJ. Using MetaMorph (Molecular Devices, Inc.), a maximum projection (comprising both highly mobile G-GGAl and unwavering (stable ) structures) and sum projection (emphasizing unwavering (stable) structures) of each stack were prepared. Each stack was thresholded (using mean +1 s.d.) and binarized. Regions 100 pixels on edge were randomly selected near the periphery of the cell and maximum projection pixels that did not overlap with the corresponding sum projection were retained, yielding an estimate of 'G-GGA1 area'. Approximately 80 regions from 20 cells for each condition in three independent experiments were analysed, and mean G-GGAl area in control cells was set to $100 \%$. Data were analysed by a two-tailed unpaired $t$-test.

Statistical analysis. Statistical analysis of directionality for Dunn chamber assays was carried out using Oriana (Kovach Computing Services, Anglesey, Wales). All other analyses were carried out using GraphPad Prism software (GraphPad Software, Inc). Parametric data were analysed using two-tailed Student $t$-tests, one-way or two-way analysis of variance, followed by Newman-Keuls or Bonferroni post hoc tests for multiple comparisons as appropriate (95\% confidence interval).

\section{References}

1. Brodsky, F. M. Diversity of clathrin function: new tricks for an old protein. Annu. Rev. Cell Dev. Biol. 28, 309-336 (2012).

2. Ybe, J. A. et al. Nuclear localization of clathrin involves a labile helix outside the trimerization domain. FEBS Lett. 587, 142-149 (2013).

3. Wilbur, J. et al. Conformation switching of clathrin light chain regulates clathrin lattice assembly. Dev. Cell 18, 841-848 (2010).

4. Hinrichsen, L., Harborth, J., Andrees, L., Weber, K. \& Ungewickell, E. J. Effect of clathrin heavy chain- and alpha -adaptin specific small interfering RNAs on endocytic accessory proteins and receptor trafficking in HeLa cells. J. Biol. Chem. 278, 45160-45170 (2003).

5. Huang, F., Khvorova, A., Marshall, W. \& Sorkin, A. Analysis of clathrin-mediated endocytosis of epidermal growth factor receptor by RNA interference. J. Biol. Chem. 279, 16657-16661 (2004).
6. Poupon, V. et al. Clathrin light chains function in mannose phosphate receptor trafficking via regulation of actin assembly. Proc. Natl Acad. Sci. USA 105, 168-173 (2008).

7. Ferreira, F. et al. Endocytosis of G protein-coupled receptors is regulated by clathrin light chain phosphorylation. Curr. Biol. 22, 1361-1370 (2012).

8. Bonazzi, M. et al. Clathrin phosphorylation is required for actin recruitment at sites of bacterial adhesion and internalization. J. Cell Biol. 195, 525-536 (2011).

9. Cureton, D. K., Massol, R. H., Whelan, S. P. J. \& Kirchhausen, T. The length of vesicular stomatitis virus particles dictates a need for actin assembly during clathrin-dependent endocytosis. PLoS Pathog. 6, e1001127 (2010).

10. Boulant, S., Kural, C., Zeeh, J. C., Ubelmann, F. \& Kirchhausen, T. Actin dynamics counteract membrane tension during clathrin-mediated endocytosis. Nat. Cell Biol. 13, 1124-1131 (2011).

11. Boettner, D. R., Friesen, H., Andrews, B. \& Lemmon, S. K. Clathrin light chain directs endocytosis by influencing the binding of the yeast Hip1R homologue, Sla2, to F-actin. Mol. Biol. Cell 22, 3699-3714 (2011).

12. Aghamohammadzadeh, S. \& Ayscough, K. R. Differential requirements for actin during yeast and mammalian endocytosis. Nat. Cell Biol. 11, 1039-1042 (2009).

13. Chen, C. Y. \& Brodsky, F. M. Huntingtin-interacting protein 1 (Hip1) and Hip1-related protein (Hip1R) bind the conserved sequence of clathrin light chains and thereby influence clathrin assembly in vitro and actin distribution in vivo. J. Biol. Chem. 280, 6109-6117 (2005).

14. Legendre-Guillemin, V. et al. Huntingtin interacting protein 1 (HIP1) regulates clathrin assembly through direct binding to the regulatory region of the clathrin light chain. J. Biol. Chem. 280, 6101-6108 (2005).

15. Gottfried, I., Ehrlich, M. \& Ashery, U. The Sla2p/HIP1/HIP1R family: similar structure, similar function in endocytosis? Biochem. Soc. Trans. 38, 187-191 (2010).

16. Wilbur, J. D. et al. Actin binding by Hip1 (huntingtin-interacting protein 1) and Hip1R (Hip1-related protein) is regulated by clathrin light chain. J. Biol. Chem. 283, 32870-32879 (2008).

17. Jones, M. C., Caswell, P. T. \& Norman, J. C. Endocytic recycling pathways: emerging regulators of cell migration. Curr. Opin. Cell Biol. 18, 549-557 (2006)

18. Ezratty, E. J., Bertaux, C., Marcantonio, E. E. \& Gundersen, G. G. Clathrin mediates integrin endocytosis for focal adhesion disassembly in migrating cells. J. Cell Biol. 187, 733-747 (2009).

19. Teckchandani, A. et al. Quantitative proteomics identifies a Dab2/integrin module regulating cell migration. J. Cell Biol. 186, 99-111 (2009).

20. Bridgewater, R. E., Norman, J. C. \& Caswell, P. T. Integrin trafficking at a glance. J. Cell Sci. 125, 3695-3701 (2012).

21. Arjonen, A., Alanko, J., Veltel, S. \& Ivaska, J. Distinct recycling of active and inactive betal integrins. Traffic 13, 610-625 (2012).

22. Shieh, J. C., Schaar, B. T., Srinivasan, K., Brodsky, F. M. \& McConnell, S. K. Endocytosis regulates cell soma translocation and the distribution of adhesion proteins in migrating neurons. PLoS ONE 6, e17802 (2011).

23. Saffarian, S., Cocucci, E. \& Kirchhausen, T. Distinct dynamics of endocytic clathrin-coated pits and coated plaques. PLoS Biol. 7, e1000191 (2009).

24. Nishimura, T. \& Kaibuchi, K. Numb controls integrin endocytosis for directional cell migration with aPKC and PAR-3. Dev. Cell 13, 15-28 (2007).

25. Zech, T. et al. The Arp2/3 activator WASH regulates alpha5beta1-integrinmediated invasive migration. J. Cell Sci. 124, 3753-3759 (2011).

26. Zhao, Y. \& Keen, J. H. Gyrating clathrin: highly dynamic clathrin structures involved in rapid receptor recycling. Traffic. 9, 2253-2264 (2008).

27. Hsu, V. W., Bai, M. \& Li, J. Getting active: protein sorting in endocytic recycling. Nat. Rev. Mol. Cell Biol. 13, 323-328 (2012).

28. Parachoniak, C. A., Luo, Y., Abella, J. V., Keen, J. H. \& Park, M. GGA3 functions as a switch to promote Met receptor recycling, essential for sustained ERK and cell migration. Dev. Cell 20, 751-763 (2011).

29. Gautier, J. J. et al. Clathrin is required for Scar/Wave-mediated lamellipodium formation. J. Cell Sci. 124, 3414-3427 (2011).

30. Foraker, A. B. et al. Clathrin promotes centrosome integrity in early mitosis through stabilization of centrosomal ch-TOG. J. Cell Biol. 198, 591-605 (2012).

31. Vassilopoulos, S. et al. A role for the CHC22 clathrin heavy-chain isoform in human glucose metabolism. Science 324, 1192-1196 (2009).

32. Esk, C., Chen, C. Y., Johannes, L. \& Brodsky, F. M. The clathrin heavy chain isoform CHC22 functions in a novel endosomal sorting step. J. Cell Biol. 188, 131-144 (2010).

33. Hynes, R. O. Integrins: bidirectional, allosteric signaling machines. Cell 110, 673-687 (2002).

34. Luo, Y., Zhan, Y. \& Keen, J. H. Arf6 regulation of gyrating-clathrin. Traffic 14, 97-106 (2013).

35. Engqvist-Goldstein, A. E. et al. The actin-binding protein Hip1R associates with clathrin during early stages of endocytosis and promotes clathrin assembly in vitro. J. Cell Biol. 154, 1209-1223 (2001).

36. Rappoport, J. Z. \& Simon, S. M. Real-time analysis of clathrin-mediated endocytosis during cell migration. J. Cell Sci. 116, 847-855 (2003). 
37. Rappoport, J. Z., Benmerah, A. \& Simon, S. M. Analysis of the AP-2 adaptor complex and cargo during clathrin-mediated endocytosis. Traffic 6, 539-547 (2005).

38. King, A. \& Loke, Y. W. Placental vascular remodelling. Lancet 350, 220-221 (1997).

39. Apps, R. et al. Genome-wide expression profile of first trimester villous and extravillous human trophoblast cells. Placenta 32, 33-43 (2011).

40. Moffett-King, A. Natural killer cells and pregnancy. Nat. Rev. Immunol. 2, 656-663 (2002).

41. Zech, T., Calaminus, S. D. \& Machesky, L. M. Actin on trafficking: could actin guide directed receptor transport? Cell Adh. Migr 6, 476-481 (2012).

42. Ybe, J. A. et al. Clathrin self-assembly is regulated by three light chain residues controlling the formation of critical salt bridges. EMBO J. 17, 1297-1303 (1998).

43. Boettner, D. R., Chi, R. J. \& Lemmon, S. K. Lessons from yeast for clathrin-mediated endocytosis. Nat. Cell Biol. 14, 2-10 (2012).

44. Bonazzi, M. et al. A common clathrin-mediated machinery co-ordinates cell-cell adhesion and bacterial internalization. Traffic 13, 1653-1666 (2012).

45. Serio, A. W., Jeng, R. L., Haglund, C. M., Reed, S. C. \& Welch, M. D. Defining a core set of actin cytoskeletal proteins critical for actin-based motility of Rickettsia. Cell Host Microbe 7, 388-398 (2010).

46. Humphries, A. C. et al. Clathrin potentiates vaccinia-induced actin polymerization to facilitate viral spread. Cell Host Microbe 12, 346-359 (2012).

47. Anitei, M. et al. Protein complexes containing CYFIP/Sra/PIR121 coordinate Arf1 and Rac1 signalling during clathrin-AP-1-coated carrier biogenesis at the TGN. Nat. Cell Biol. 12, 330-340 (2010).

48. Baird, G. S., Zacharias, D. A. \& Tsien, R. Y. Biochemistry, mutagenesis, and oligomerization of DsRed, a red fluorescent protein from coral. Proc. Nat. Acad. Sci. USA 97, 11984-11989 (2000).

49. Brodsky, F. M. \& Parham, P. Polymorphism in clathrin light chains from different tissues. J. Mol. Biol. 167, 197-204 (1983).

50. Acton, S. L. \& Brodsky, F. M. Predominance of clathrin light chain LCb correlates with the presence of a regulated secretory pathway. J. Cell Biol. 111, 1419-1426 (1990).

51. Rao, D. S. et al. Huntingtin-interacting protein 1 is overexpressed in prostate and colon cancer and is critical for cellular survival. J. Clin. Invest. 110, 351-360 (2002).

52. Bradley, S. V. et al. Aberrant Huntingtin interacting protein 1 in lymphoid malignancies. Cancer Res. 67, 8923-8931 (2007).

53. Rao, D. S. et al. Altered receptor trafficking in Huntingtin Interacting Protein 1-transformed cells. Cancer Cell 3, 471-482 (2003).

54. Brodsky, F. M. Clathrin structure characterized with monoclonal antibodies. I. Analysis of multiple antigenic sites. J. Cell Biol. 101, 2047-2054 (1985).
55. Acton, S. L., Wong, D. H., Parham, P., Brodsky, F. M. \& Jackson, A. P. Alteration of clathrin light chain expression by transfection and gene disruption. Mol. Biol. Cell 4, 647-660 (1993).

56. Näthke, I. et al. Folding and trimerization of clathrin subunits at the triskelion hub. Cell 68, 899-910 (1992).

57. Brodsky, F. M. et al. Localization of clathrin light-chain sequences mediating heavy-chain binding and coated vesicle diversity. Nature 326, 203-205 (1987).

58. Smyth, G. K. Linear models and empirical bayes methods for assessing differential expression in microarray experiments. Stat. Appl. Genet. Mol. Biol. 3, Article3 (2004).

\section{Acknowledgements}

This work was supported by NIH grant GM038093 to F.M.B. and GM049217 to J.H.K., and NIH training grants T32 GM07175 and NCI F31 CA171594 to S.R.M.

\section{Author contributions}

S.R.M., L.V., C-Y.C., A.M., J.H.K. and F.M.B. designed the experiments. S.R.M., L.V., C-Y.C., Y.L., J.A.T., T.M.E., A.S., A.B.F., N.M.L.W. and C.E. performed the experiments A.M. contributed sample material and T.A.F. contributed analytical tools. S.R.M. and F.M.B. wrote the manuscript with input from L.V., C-Y.C., Y.L., A.M. and J.H.K.

\section{Additional information}

Supplementary Information accompanies this paper at http://www.nature.com/ naturecommunications

Competing financial interests: The authors declare no competing financial interests.

Reprints and permission information is available online at http://npg.nature.com/ reprintsandpermissions/

How to cite this article: Majeed, S. R. et al. Clathrin light chains are required for the gyrating-clathrin recycling pathway and thereby promote cell migration. Nat. Commun. 5:3891 doi: 10.1038/ncomms4891 (2014).

(c) (1) $\Theta$ This work is licensed under a Creative Commons AttributionNonCommercial-NoDerivs 3.0 Unported License. The images or other third party material in this article are included in the article's Creative Commons license, unless indicated otherwise in the credit line; if the material is not included under the Creative Commons license, users will need to obtain permission from the license holder to reproduce the material. To view a copy of this license, visit http://creativecommons. org/licenses/by-nc-nd/3.0/ 